\title{
Improving Productivity and Fruit Quality of Florida Prince Peach Trees by Using Some Agriculture Treatments
}

\author{
M. A. Zayan, S. M. Zeerban, G. B. Mikhael* and H. M. \\ Abo Ogiela" \\ Pomology Department, Faculty of Agriculture, Kafrelsheikh University, \\ Kafrelsheikh and "Deciduous Fruit Tree. Dep., Horticulture Research \\ Institute, Agricultural Research Centre, Cairo, Egypt.
}

7 HIS investigation was carried out during 2009 and 2010 seasons on eight years old peach trees grown in a commercial orchard located at Sedy Salem District, Kafr El-Sheikh governorate. The effects of thinning out and heading back pruning, fruit thinning and their interaction on improving yield and fruit quality of Florida Prince peach cultivar specially fruit size and colour were studied. Thinning out and heading back pruning treatments and hand fruit thinning levels revealed significant variation in yield and fruit quality of Florida Prince peach trees. Therefore, the interaction (TO x HB x FT) which was significant in most cases exhibited the most important data in the present work. Thus, thinning out $50 \%$ of the number of one year old shoot and heading back $25 \%$ from the length of one year old shoot with fruit thinning at $15 \mathrm{~cm}$ apart, considered the best combination treatment. This treatment produced maximum yield as $\mathrm{kg} / \mathrm{tree}$, the highest number and percentage of large sized fruit with high quality specially fruit weight, size, colour and its content of TSS, vitamin C and anthocy anin.

Keywords: Florida Prince, Pruning, Yield, Fruit characteristics.

Peach is one of the most important deciduous fruit trees grown in Egypt. The total planted area increased rapidly through the last three decades due to introduced several peach cultivars of low and moderate chilling requirements by the Agricultural Development system (Stino et al., 1982 and Mansour \& Stino, 1986a, 1986b). It reached about 80609 feddans with a production of about 273156 tons according to the last statistics of Ministry of Agriculture and Land Reclamation (2013). Fruit size and colour are the major criterion of peach fruit quality since pruning and fruit thinning are considered the two agricultural practices that affected fruit size and colour (Zayan, 1991 and Eliwa, 2003). Pruning is an essential cultural practice in the production of peaches. As trees aged, pruning stimulate new growth and provides essential light distribution through the tree for the formation of large fruit with acceptable fruit quality. Appropriate fruit colour, soluble solids and ripeness. Pruning can be used to judiciously remove a significant portion of the unwanted potential crop at a lower cost than hand thinning (Li et al., 2003 and Fumey et al., 2008). Fruit thinning is usually performed in peach orchards in order to improve fruit size (Corelli-Grappadelli and Costen, 1991). The principal aim of thinning is to optimize the leaf to fruit ratio (Sansavini et al., 1985). Furthermore, hand 
thinning is certainly the most accurate method, which allowed space fruit regularly along a branch at about specific space. However, it is considered more profitable to select large and well formed fruits and eliminate smaller and deformed ones. These later seldom achieve good quality at harvest (Southwick et al., 1995 and Eliwa, 2003). The objective of this experiment was to study the possible effects of thinning out, heading back, fruit thinning and their interaction on yield and fruit quality of "Florida Prince" peach trees.

\section{Materials and Methods}

The present study was carried out during two successive season of 2009 and 2010 on eight years old Florida Prince peach cv. trees (Prunus persica L. Batsch) and grown in private orchard located at Sedy Salem district, Kafrelsheikh Governorate. Trees were subjected to horticulture practices usually done in this region.

At winter pruning (15 November), three degrees of thinning out pruning were carried out by removing 25,50 and $75 \%$ of one year old shoots $\left(\mathrm{To}, \mathrm{To}_{2}, \mathrm{To}_{3}\right)$.

Also, three degrees of heading back pruning were applied by removing $25 \%$ and $50 \%$ of length of each one year-old shoot corresponding to HB1 (unpruned), HB2 (light heading back)and HB3 (severe heading back), respectively.

Hand fruit thinning was carried out after fruit set by leaving one fruit for 10 and $15 \mathrm{~cm}$ apart on fruiting shoots The tree level of thinning out pruning (To1, $\left.\mathrm{To}_{2}, \mathrm{To}_{3}\right)$ and the three degrees of heading back pruning (HB1, HB2, and HB3) as well as the two levels of fruit thinning (FT1 and FT2) were arranged in 18 combination treatments ( 3 thinning out $\times 3$ heading back $\times 2$ fruit thinning). All combination treatments used in this experiment are listed in Table 1.

TABLE 1. Treatment .

\begin{tabular}{|c|c|c|}
\hline Thinning out (TO) & Heading back (HB) & Fruit thinning (FT) \\
\hline \multirow{6}{*}{ Thinning out $25 \%$ (To1) } & \multirow{2}{*}{ Heading Back 0\% (HB1) } & Fruit thinning at $10 \mathrm{~cm}(\mathrm{FT} 1)^{*}$ \\
\hline & & Fruit thinning at $15 \mathrm{~cm}(\mathrm{FT} 2)$ \\
\hline & \multirow{2}{*}{ Heading Back 25\% (HB2) } & Fruit thinning at $10 \mathrm{~cm}(\mathrm{FT})$ \\
\hline & & Fruit thinning at $15 \mathrm{~cm}(\mathrm{FT} 2)$ \\
\hline & \multirow{2}{*}{ Heading Back 50\% (HB3) } & Fruit thinning at $10 \mathrm{~cm}(\mathrm{FT})$ \\
\hline & & Fruit thinning at $15 \mathrm{~cm}(\mathrm{FT} 2)$ \\
\hline \multirow{6}{*}{ Thinning out $50 \%$ (To2) } & \multirow{2}{*}{ Heading Back 0\% (HB1) } & Fruit thinning at $10 \mathrm{~cm}$ (FT1) \\
\hline & & Fruit thinning at $15 \mathrm{~cm}(\mathrm{FT} 2)$ \\
\hline & \multirow{2}{*}{ Heading Back 25\% (HB2) } & Fruit thinning at $10 \mathrm{~cm}(\mathrm{FT} 1)$ \\
\hline & & Fruit thinning at $15 \mathrm{~cm}(\mathrm{FT} 2)$ \\
\hline & \multirow{2}{*}{ Heading Back 50\% (HB3) } & Fruit thinning at $10 \mathrm{~cm} \mathrm{(FT1)}$ \\
\hline & & Fruit thinning at $15 \mathrm{~cm}(\mathrm{FT} 2)$ \\
\hline \multirow{6}{*}{ Thinning out $75 \%$ (To3) } & \multirow{2}{*}{ Heading Back 0\% (HB1) } & Fruit thinning at $10 \mathrm{~cm}(\mathrm{FT} 1)$ \\
\hline & & Fruit thinning at $15 \mathrm{~cm}(\mathrm{FT} 2)$ \\
\hline & \multirow{2}{*}{ Heading Back 25\% (HB2) } & Fruit thinning at $10 \mathrm{~cm}(\mathrm{FT})$ \\
\hline & & Fruit thinning at $15 \mathrm{~cm}$ (FT2) \\
\hline & \multirow[t]{2}{*}{ Heading Back 50\% (HB3) } & Fruit thinning at $10 \mathrm{~cm}$ (FT1) \\
\hline & & Fruit thinning at $15 \mathrm{~cm}$ (FT2) \\
\hline
\end{tabular}

This treatment served as control

Egypt. J. Hort. Vol. 42, No.1 (2015) 
A randomized complete block design as a factorial experiment was used. The obtained data were subjected to statistical analysis according to Snedecor and Cochran (1990). The LSD test at 0.5 and 0.1 level was used to compare between the means.

Measurements and Determinations

Yield and its components

Tree fruit yield was divided into 3 classes according to fruit size i.e. (->5.5 $\mathrm{cm}),(5.5-6.0 \mathrm{~cm})$ and $(6.0>-\mathrm{cm})$. Number and percent of fruit of each class were also recorded. Yield per tree was recorded as number and weight $\mathrm{kg} / \mathrm{tree}$. Yield efficiency (YE) as fruit weight $\mathrm{kg}$ per $\mathrm{cm}^{2}$ of trunk cross section area (TCSA) was estimated.

Fruit quality

At harvest time (April $5^{\text {th }}$ and April $6^{\text {th }}$ ) in 2009 and 2010 season, ten fruit were selected at random from each tree and prepared for the determination of physical and chemical fruit characteristics.

\section{Physical fruit quality:}

Fruit weight $(\mathrm{g})$, length and diameter $(\mathrm{cm})$ were measured and their fruit shapes (LD) ratio were calculated. Fruit volume in ml was determined by water displacement. A Magness-Taylor type pressure tester with plunger of 5/16 inch ${ }^{2}$ was used for determining flesh fruit firmness $\left(\mathrm{lb} / \mathrm{in}^{2}\right)$. Fruit colour was visually determined for each fruit sample according to colour degree expressed on number as follows:

$0=$ green colour and $10=$ deep red

Chemical fruit quality

Soluble solids contents (TSS), total acidity, TSS/acidity ratio ascorbic acid (VC) as $\mathrm{mg} / 100 \mathrm{~g}$ fresh weight was determined according to A.O.A.C. (1990).Total anthocyanin: measured according to Hsia et al. (1965).

\section{Yield}

\section{Results and Discussion}

Number of fruit per tree

Data in Tables 2 and 3 revealed that number of fruits/tree was significantly reduced by increasing the severity of thinning out and heading back pruning treatments. This effect may be due to the effect of dormant thinning out and heading back in reducing the number of flowers per bearing shoot (Mikhael, 2001).

These results herein are in line with those obtained by Zayan (1991) and Mikhael et al. (2012) working on "Dessert Red" peach trees mentioned that, severe pruned trees (thinning out 50\%) produced the least number of fruit per tree. As for the effect of hand thinning, it is clear that, fruit thinning at $15 \mathrm{~cm}$ apart of bearing shoot significantly reduced the total number of fruits per tree compared to fruit thinning at $10 \mathrm{~cm}$ apart, in both seasons. Similar results were obtained by said et al. (2003), Nijorog and Reighard (2008) and Mohsen (2010). 
However, the interaction was significant in both seasons and the highest number of fruits belonged to the control treatment (To1 x HB1 x FT1) with (510 and 440) in 2009 and 2010 seasons, respectively whereas (To3x HB3 x FT2) combination treatment gave the least fruit number per tree (236 and 216) in both seasons, respectively.

\section{Yield ( $\mathrm{kg} /$ tree $)$}

Data in Tables 2 and 3 exhibited that moderate thinning out treatment (50\%) recorded the highest yield compared to light and severe ones (25 and 75\%). However, severe treatments produced the least yield $(\mathrm{kg} / \mathrm{tree})$ in both seasons. Concerning the impact of heading back treatments, the data disclosed that, light headed trees $(25 \%)$ produced maximum yield in both seasons. Meanwhile, severe headed trees (50\%) gave minimum yield ( $\mathrm{kg} /$ tree) when compared to un-headed ones (control). Similar effect was obtained by Rathi et al. (2003) on "Tessia Samisto" peach, Siham et al. (2005) on "Alexandra" peach and Mikhael et al. (2012) on Dessert Red peach cvs . The data also clarify no significantly differences were found in tree yield $(\mathrm{kg})$ between the two tested fruit thinning treatments at 10 and $15 \mathrm{~cm}$, in both seasons. These findings are in accordance with those obtained by Egea et al. (1989) and Myer et al. (1993), Nijorog and Reighard (2008) and Mohsen (2010) on peach cvs., they indicated that hand fruit thinning treatments reduced the yield as weight of fruits $(\mathrm{kg} / \mathrm{tree})$.However, the most important effect was obtained by the interaction which was significant in both seasons and the highest yield (kg/tree) came from (To2 x HB2 x FT2) and (To2 x HB2 x FT1) combination treatments without significant differences between them. While the least yield ( $\mathrm{kg} /$ tree) was always belonged to (To3 $\mathrm{x}$ HB3 x FT2) treatment in both seasons.

Yield efficiency (YE) $\left(\mathrm{kg} / \mathrm{cm}^{2}\right)$ TCSA

As shown in Tables 2 and 3, data of both seasons disclosed that yield efficiency (YE) determined as $\mathrm{kg} / \mathrm{cm}^{2}$ of trunk cross section area take the same trend of yield (kg/tree) as influenced by thinning out, heading back, fruit thinning and their interaction.

These results are in agreement with those reported by Mikhael et al. (2012), Davarynefad et al. (2008) and Reginoto et al. (1995) which they mentioned that yield efficiency was decreased by thinning ten year old "fairland" nectarine trees at 15 days before pit hardening to normal density 2.5 fruit $/ \mathrm{cm}^{2}$ TCSA. However, the interaction (To $\mathrm{x}$ HB $\mathrm{x}$ FT) was significant in both season and the highest values always belonged to (To2 x HB2 x FT1) and (TO2 x HB2 x FT2) combination treatments without significant differences between them in both seasons.

Egypt. J. Hort. Vol. 42, No.1 (2015) 
TABLE 2. Effect of thinning out, heading back, fruit thinning and their interaction on yield of "Florida Prince" peach trees in 2009 season.

\begin{tabular}{|c|c|c|c|c|c|c|c|c|}
\hline \multirow{2}{*}{\multicolumn{3}{|c|}{ Treatment }} & \multicolumn{4}{|c|}{ Yield } & \multirow{2}{*}{\multicolumn{2}{|c|}{$\begin{array}{l}\text { Yield efficiency } \\
\left(\mathrm{kg} / \mathrm{cm}^{2}\right)\end{array}$}} \\
\hline & & & \multicolumn{2}{|c|}{ No. of fruits/tree } & \multicolumn{2}{|c|}{ kg/tree } & & \\
\hline \multirow{3}{*}{$\begin{array}{l}\text { Thinning } \\
\text { out (To) }\end{array}$} & \multirow{3}{*}{\multicolumn{2}{|c|}{$\begin{array}{l}\text { Heading back } \\
\text { (HB) }\end{array}$}} & Fruit & Fruit & Fruit & Fruit & Fruit & Fruit \\
\hline & & & thinning & thinning & thinning & thinning & thinning & thinning \\
\hline & & & $\begin{array}{l}10 \mathrm{~cm} \\
\text { (FT1) }\end{array}$ & $\begin{array}{l}15 \mathrm{~cm} \\
\text { (FT2) }\end{array}$ & $\begin{array}{l}10 \mathrm{~cm} \\
\text { (FT1) }\end{array}$ & $\begin{array}{l}15 \mathrm{~cm} \\
\text { (FT2) }\end{array}$ & $\begin{array}{l}10 \mathrm{~cm} \\
\text { (FT1) }\end{array}$ & $\begin{array}{l}15 \mathrm{~cm} \\
\text { (FT2) }\end{array}$ \\
\hline \multirow{3}{*}{ To1 } & & HB1 & 510 & 424 & 39.84 & 38.77 & 0.375 & 0.357 \\
\hline & & HB2 & 469 & 398 & 41.16 & 41.09 & 0.356 & 0.378 \\
\hline & & HB3 & 394 & 327 & 36.19 & 35.01 & 0.326 & 0.321 \\
\hline \multirow{3}{*}{ То2 } & & HB1 & 490 & 422 & 41.72 & 39.89 & 0.397 & 0.374 \\
\hline & & HB2 & 478 & 387 & 44.86 & 45.60 & 0.412 & 0.426 \\
\hline & & HB3 & 356 & 284 & 34.87 & 33.77 & 0.309 & 0.310 \\
\hline \multirow{3}{*}{ To3 } & & HB1 & 384 & 308 & 33.68 & 32.95 & 0.330 & 0.297 \\
\hline & & HB2 & 374 & 297 & 35.84 & 34.59 & 0.335 & 0.307 \\
\hline & & HB3 & 278 & 236 & 28.17 & 27.91 & 0.251 & 0.247 \\
\hline \multicolumn{3}{|c|}{ L.S.D. interaction 0.05} & \multicolumn{2}{|c|}{15.7} & \multicolumn{2}{|c|}{2.520} & \multicolumn{2}{|c|}{0.0309} \\
\hline \multicolumn{3}{|c|}{0.01} & \multicolumn{2}{|c|}{21.1} & \multicolumn{2}{|c|}{3.383} & \multicolumn{2}{|c|}{0.0416} \\
\hline \multirow{3}{*}{\multicolumn{2}{|c|}{$\begin{array}{l}\text { Mean effect of } \\
\text { thinning out }\end{array}$}} & \begin{tabular}{|c|} 
Thinning out \\
$25 \%$ \\
\end{tabular} & \multicolumn{2}{|c|}{420} & \multicolumn{2}{|c|}{38.71} & \multicolumn{2}{|c|}{0.352} \\
\hline & & \begin{tabular}{|c|} 
Thinning out \\
$50 \%$
\end{tabular} & \multicolumn{2}{|c|}{403} & \multicolumn{2}{|c|}{40.12} & \multicolumn{2}{|c|}{0.372} \\
\hline & & $\begin{array}{c}\text { Thinning out } \\
75 \% \\
\end{array}$ & \multicolumn{2}{|c|}{312} & \multicolumn{2}{|c|}{32.19} & \multicolumn{2}{|c|}{0.295} \\
\hline \multicolumn{3}{|c|}{ L.S.D. 0.05} & \multicolumn{2}{|c|}{6.4} & & 29 & 0.01 & 126 \\
\hline & S.D & 0.01 & 8. & 6 & & 381 & 0.01 & 170 \\
\hline $\begin{array}{l}\text { Mean effe } \\
\text { heading } b\end{array}$ & $\begin{array}{l}\mathrm{t} \text { of } \\
\mathrm{ck}\end{array}$ & $\begin{array}{c}\text { Heading back } \\
0 \% \\
\end{array}$ & 42 & 23 & & .81 & 0.3 & 55 \\
\hline & & $\begin{array}{c}\text { Heading back } \\
25 \%\end{array}$ & 40 & 01 & & .56 & 0.3 & 69 \\
\hline & & $\begin{array}{c}\text { Heading back } \\
50 \% \\
\end{array}$ & 31 & 13 & & .65 & 0.2 & 94 \\
\hline & S.D & 0.05 & 6. & 4 & & 29 & 0.01 & 126 \\
\hline & S.D & 0.01 & 8. & 6 & & 381 & 0.01 & 170 \\
\hline $\begin{array}{l}\text { Mean effe } \\
\text { fruit thinn }\end{array}$ & of & \begin{tabular}{|c|}
$\begin{array}{c}\text { Fruit thinning } \\
10 \mathrm{~cm}\end{array}$ \\
\end{tabular} & 41 & 15 & & .37 & 0.3 & 44 \\
\hline & & $\begin{array}{c}\text { Fruit thinning } \\
15 \mathrm{~cm}\end{array}$ & 34 & 43 & & .64 & 0.3 & 35 \\
\hline & S.D & 0.05 & 5. & 2 & & JS & $\mathrm{N}$ & S \\
\hline & S.D & 0.01 & 7. & 0 & & IS & $\mathrm{N}$ & $S$ \\
\hline
\end{tabular}

Egypt. J. Hort. Vol. 42, No.1 (2015) 
TABLE 3. Effect of thinning out, heading back, fruit thinning and their interaction on yield of "Florida Prince" peach trees in 2010 season.

\begin{tabular}{|c|c|c|c|c|c|c|c|}
\hline \multirow{2}{*}{\multicolumn{2}{|c|}{ Treatment }} & \multicolumn{4}{|c|}{ Yield } & \multirow{2}{*}{\multicolumn{2}{|c|}{$\begin{array}{l}\text { Yield efficiency } \\
\left(\mathrm{kg} / \mathrm{cm}^{2}\right)\end{array}$}} \\
\hline & & \multicolumn{2}{|c|}{ No. of fruits/tree } & \multicolumn{2}{|c|}{ kg/tree } & & \\
\hline $\begin{array}{l}\text { Thinning } \\
\text { out (To) }\end{array}$ & $\begin{array}{c}\text { Heading } \\
\text { back } \\
(\mathrm{HB})\end{array}$ & $\begin{array}{c}\text { Fruit } \\
\text { thinning } \\
10 \mathrm{~cm} \\
\text { (FT1) }\end{array}$ & $\begin{array}{c}\text { Fruit } \\
\text { thinning } \\
15 \mathrm{~cm} \\
\text { (FT2) }\end{array}$ & $\begin{array}{c}\text { Fruit } \\
\text { thinning } \\
10 \mathrm{~cm} \\
\text { (FT1) }\end{array}$ & $\begin{array}{c}\text { Fruit } \\
\text { thinning } \\
15 \mathrm{~cm} \\
\text { (FT2) }\end{array}$ & $\begin{array}{c}\text { Fruit } \\
\text { thinning } \\
10 \mathrm{~cm} \\
\text { (FT1) }\end{array}$ & $\begin{array}{c}\text { Fruit } \\
\text { thinning } \\
15 \mathrm{~cm} \\
\text { (FT2) }\end{array}$ \\
\hline \multirow{3}{*}{ To1 } & HB1 & 440 & 381 & 36.24 & 36.35 & 0.327 & 0.322 \\
\hline & HB2 & 429 & 351 & 37.93 & 37.65 & 0.336 & 0.340 \\
\hline & HB3 & 331 & 282 & 31.32 & 30.86 & 0.288 & 0.283 \\
\hline \multirow{3}{*}{ Тo2 } & HB1 & 427 & 379 & 37.70 & 37.09 & 0.340 & 0.334 \\
\hline & HB2 & 422 & 339 & 40.89 & 40.93 & 0.378 & 0.383 \\
\hline & HB3 & 324 & 262 & 32.65 & 31.68 & 0.295 & 0.272 \\
\hline \multirow{3}{*}{ To3 } & HB1 & 346 & 284 & 31.58 & 30.83 & 0.280 & 0.283 \\
\hline & HB2 & 342 & 275 & 33.71 & 32.62 & 0.298 & 0.293 \\
\hline & HB3 & 253 & 216 & 26.59 & 26.04 & 0.228 & 0.235 \\
\hline \multicolumn{2}{|c|}{ L.S.D. interaction 0.05} & \multicolumn{2}{|c|}{16.0} & \multicolumn{2}{|c|}{2.576} & \multicolumn{2}{|c|}{0.0204} \\
\hline \multicolumn{2}{|c|}{0.01} & \multicolumn{2}{|c|}{21.5} & \multicolumn{2}{|c|}{3.459} & \multicolumn{2}{|c|}{0.0275} \\
\hline \multirow{3}{*}{$\begin{array}{l}\text { Mean } \\
\text { effect of } \\
\text { thinning } \\
\text { out }\end{array}$} & $\begin{array}{l}\text { Thinning } \\
\text { out } 25 \%\end{array}$ & \multicolumn{2}{|c|}{369} & \multicolumn{2}{|c|}{35.06} & \multicolumn{2}{|c|}{0.316} \\
\hline & $\begin{array}{l}\text { Thinning } \\
\text { out } 50 \%\end{array}$ & \multicolumn{2}{|c|}{359} & \multicolumn{2}{|c|}{36.82} & \multicolumn{2}{|c|}{0.334} \\
\hline & $\begin{array}{l}\text { Thinning } \\
\text { out } 75 \%\end{array}$ & \multicolumn{2}{|c|}{286} & \multicolumn{2}{|c|}{30.23} & \multicolumn{2}{|c|}{0.270} \\
\hline \multicolumn{2}{|c|}{ L.S.D. 0.05} & \multicolumn{2}{|c|}{6.5} & \multicolumn{2}{|c|}{1.052} & \multicolumn{2}{|c|}{0.0083} \\
\hline \multicolumn{2}{|c|}{ L.S.D. 0.01} & & & & & & \\
\hline $\begin{array}{l}\text { Mean } \\
\text { effect of }\end{array}$ & $\begin{array}{l}\text { Heading } \\
\text { back 0\% }\end{array}$ & & & & & & \\
\hline $\begin{array}{l}\text { heading } \\
\text { back }\end{array}$ & $\begin{array}{c}\text { Heading } \\
\text { back } \\
25 \%\end{array}$ & & & & & & \\
\hline & $\begin{array}{l}\text { Heading } \\
\text { back } \\
50 \%\end{array}$ & & & & & & \\
\hline$\overline{\text { L.S.D }}$ & 0.05 & & & & & & \\
\hline L.S.D & 0.01 & & & & & & \\
\hline $\begin{array}{l}\text { Mean } \\
\text { effect of } \\
\text { fruit }\end{array}$ & $\begin{array}{c}\text { Fruit } \\
\text { thinning } \\
10 \mathrm{~cm}\end{array}$ & & & & & & \\
\hline thinning & $\begin{array}{l}\text { Fruit } \\
\text { thinning } \\
15 \mathrm{~cm}\end{array}$ & & & & & & \\
\hline L.S.D & 0.05 & & & & & & \\
\hline L.S.D & 0.01 & & & & & & \\
\hline
\end{tabular}

Egypt. J. Hort. Vol. 42, No.1 (2015) 
Fruit size and percentage of large fruits

Data presented in Tables 4 and 5 exhibited that, the interaction was significant in both seasons and (To $2 \times$ HB2 $x$ FT2) combination treatment produced the highest number and percentage of large fruits $(6.0>\mathrm{cm}$ diameter $)$ in both seasons,. Concerning the effect of fruit thinning, the data revealed that increasing the space between fruits from 10 to $15 \mathrm{~cm}$ apart significantly increased the number and percentage of large fruits but reduced the number and percent of medium and small fruit in both seasons. The obtained results are in line with those obtained by Abdel-Hamid (1998) and Eliwa (2003) who found thathand thinning increased yield \% in the first picking and large fruit ( $>90 \mathrm{~g}$ ) of "Mit Ghamr" peach when compared to the control.

TABLE 4. Effect of thinning out, heading back, fruit thinning and their interaction on number and percentage of fruit size of "Florida Prince" peach trees in 2009 season.

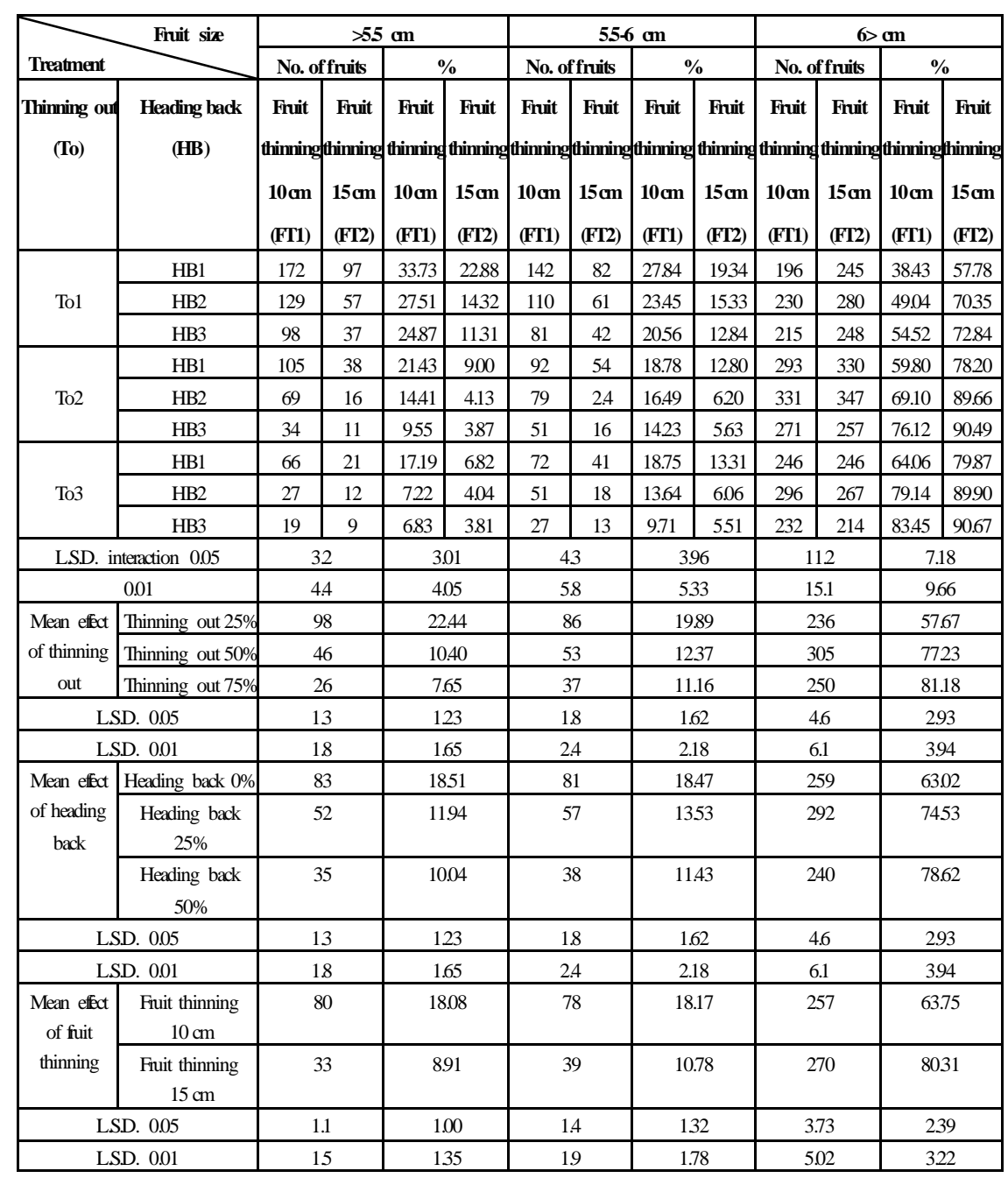

Egypt. J. Hort. Vol. 42, No.1 (2015) 
TABLE 5. Effect of thinning out, heading back, fruit thinning and their interaction on number and percentage of fruit size classes of "Florida Prince" peach trees in 2010 season.

\begin{tabular}{|c|c|c|c|c|c|c|c|c|c|c|c|c|c|}
\hline \multirow{2}{*}{\multicolumn{2}{|c|}{ Treatment }} & \multicolumn{4}{|c|}{$>5.5 \mathrm{~cm}$} & \multicolumn{4}{|c|}{$5.5-6 \mathrm{~cm}$} & \multicolumn{4}{|c|}{$6>\mathrm{cm}$} \\
\hline & & \multicolumn{2}{|c|}{ No. of fruits } & \multicolumn{2}{|c|}{$\%$} & \multicolumn{2}{|c|}{ No. of fruits } & \multicolumn{2}{|c|}{$\%$} & \multicolumn{2}{|c|}{ No. of fruits } & \multicolumn{2}{|c|}{$\%$} \\
\hline \multirow{4}{*}{ Thinning } & Heading & Fruit & Fruit & Fruit & Fruit & Fruit & Fruit & Fruit & Fruit & Fruit & Fruit & Fruit & Fruit \\
\hline & back & thinnin & thinning & thinning & thinning & thinning & othinning & thinning & thinning & thinning & thinning & thinning & gthinning \\
\hline & (HB) & $10 \mathrm{~cm}$ & $15 \mathrm{~cm}$ & $10 \mathrm{~cm}$ & $15 \mathrm{~cm}$ & $10 \mathrm{~cm}$ & $15 \mathrm{~cm}$ & $10 \mathrm{~cm}$ & $15 \mathrm{~cm}$ & $10 \mathrm{~cm}$ & $15 \mathrm{~cm}$ & $10 \mathrm{~cm}$ & $15 \mathrm{~cm}$ \\
\hline & & (FT1) & (FT2) & (FT1) & (FT2) & (FT1) & (FT2) & (FT1) & (FT2) & (FT1) & (FT2) & (FT1) & (FT2) \\
\hline \multirow{3}{*}{ Tol } & $\mathrm{HB} 1$ & 145 & 71 & 32.95 & 18.64 & 121 & 86 & 27.50 & 22.57 & 174 & 224 & 39.55 & 58.79 \\
\hline & $\mathrm{HB} 2$ & 117 & 42 & 27.27 & 11.97 & 99 & 56 & 23.08 & 15.92 & 213 & 253 & 49.65 & 72.08 \\
\hline & $\mathrm{HB} 3$ & 82 & 25 & 24.77 & 8.87 & 63 & 39 & 19.03 & 13.83 & 186 & 218 & 56.19 & 77.30 \\
\hline \multirow{3}{*}{ T02 } & $\mathrm{HB} 1$ & 91 & 31 & 21.31 & 8.18 & 78 & 49 & 18.27 & 12.95 & 258 & 299 & 60.42 & 78.89 \\
\hline & $\mathrm{HB} 2$ & 57 & 15 & 13.51 & 4.42 & 71 & 19 & 16.82 & 5.61 & 294 & 305 & 69.67 & 8.97 \\
\hline & $\mathrm{HB} 3$ & 34 & 11 & 10.49 & 4.20 & 39 & 14 & 12.04 & 5.34 & 251 & 237 & 77.47 & 90.45 \\
\hline \multirow{3}{*}{ To3 } & $\mathrm{HB} 1$ & 51 & 24 & 14.74 & 8.45 & 68 & 31 & 19.65 & 10.92 & 227 & 229 & 65.61 & 80.63 \\
\hline & HB2 & 31 & 12 & 9.06 & 4.36 & 38 & 15 & 11.1 & 5.45 & 273 & 248 & 79.82 & 90.18 \\
\hline & $\mathrm{HB} 3$ & 18 & 9 & 7.11 & 4.17 & 22 & 12 & 8.7 & 5.56 & 213 & 195 & 84.19 & 90.28 \\
\hline \multicolumn{2}{|c|}{$\begin{array}{l}\text { L.S.D. interaction } \\
0.05\end{array}$} & \multicolumn{2}{|c|}{4.9} & \multicolumn{2}{|c|}{2.87} & \multicolumn{2}{|c|}{5.6} & \multicolumn{2}{|c|}{3.28} & \multicolumn{2}{|c|}{12.6} & \multicolumn{2}{|c|}{6.14} \\
\hline \multicolumn{2}{|c|}{0.01} & \multicolumn{2}{|c|}{6.6} & \multirow{2}{*}{\multicolumn{2}{|c|}{$\begin{array}{l}3.86 \\
20.75\end{array}$}} & \multicolumn{2}{|c|}{7.6} & \multicolumn{2}{|c|}{4.41} & \multicolumn{2}{|c|}{16.9} & & 1.26 \\
\hline $\begin{array}{c}\text { Mean } \\
\text { effect of }\end{array}$ & \begin{tabular}{|l|} 
Thinning \\
out $25 \%$
\end{tabular} & & 0 & & & & 77 & & 0.33 & & 11 & & 9.93 \\
\hline $\begin{array}{c}\text { thinning } \\
\text { out }\end{array}$ & $\begin{array}{l}\text { Thinning } \\
\text { out } 50 \%\end{array}$ & & 0 & $\overline{10}$ & $\overline{35}$ & & 45 & & 1.84 & 2 & $\overline{74}$ & & 7.81 \\
\hline & \begin{tabular}{|l|} 
Thinning \\
out $75 \%$
\end{tabular} & & 4 & 7. & 98 & & 31 & & 0.23 & 2 & 31 & & 1.79 \\
\hline L.S.D. & 0.05 & & 0 & 1. & 17 & & 2.3 & & 34 & 5 & 2 & & 1.51 \\
\hline L.S.D. & 0.01 & & 7 & 1. & 58 & & 3.1 & & 80 & 6 & 9 & & 37 \\
\hline $\begin{array}{c}\text { Mean } \\
\text { effect of }\end{array}$ & $\begin{array}{l}\text { Heading } \\
\text { back 0\% }\end{array}$ & & 9 & 17 & 1.38 & & $\overline{72}$ & & 8.64 & $\overline{2}$ & $\overline{35}$ & & $\begin{array}{l}3.98 \\
\end{array}$ \\
\hline $\begin{array}{c}\text { heading } \\
\text { back }\end{array}$ & $\begin{array}{c}\text { Heading } \\
\text { back } \\
25 \%\end{array}$ & & 6 & & 1.77 & & 50 & & 3.00 & 2 & 64 & & 5.23 \\
\hline & $\begin{array}{c}\text { Heading } \\
\text { back } \\
50 \%\end{array}$ & & 0 & 9. & 94 & & 32 & & $\overline{7.75}$ & 2 & 17 & & 9.31 \\
\hline $\begin{array}{l}\text { L.S.D. } \\
\text { L. }\end{array}$ & 0.05 & & 0 & 1. & .17 & & 2.3 & & 34 & 5 & 2 & & 1.51 \\
\hline L.S.D. & 0.01 & & 7 & 1. & 58 & & 3.1 & & 80 & 6 & 9 & & 37 \\
\hline $\begin{array}{c}\text { Mean } \\
\text { effect of } \\
\text { fruit }\end{array}$ & \begin{tabular}{|c|} 
Fruit \\
thinning \\
$10 \mathrm{~cm}$
\end{tabular} & & $\overline{0}$ & & 1.91 & & 57 & & 7.36 & $\overline{2}$ & $\overline{32}$ & & 4.73 \\
\hline thinning & $\begin{array}{c}\begin{array}{c}\text { Frit } \\
\text { thinning } \\
15 \mathrm{~cm}\end{array} \\
\end{array}$ & & 7 & & .14 & & 36 & & 0.91 & 2 & 45 & & 0.95 \\
\hline L.S.D. & 0.05 & & 6 & 0. & 96 & & 1.7 & & .09 & 4 & 2 & & 2.05 \\
\hline L.S.D. & 0.01 & & 2 & 1. & 29 & & $\sqrt{2.3}$ & & 47 & 5 & 7 & & 1.75 \\
\hline
\end{tabular}

Egypt. J. Hort. Vol. 42, No.1 (2015) 
Data also exhibited that, the highest number of large sized fruit was obtained by moderate thinning out degree (50\%) (To2) and light heading back level 25\% (HB2) compared to other levels, while the percentage of large sized fruits was linearly increased by increasing the severity of thinning out and heading back pruning. While, the number and percentage of medium and small sized fruit were decreased by increasing the severity of thinning out and heading back. The differences were significant in both seasons. These results are in complete agreement with those obtained by Zayan (1991) and Sharma et al. (2001) who revealed that severe pruned trees $(75 \%)$ produced the highest percentage of large size fruits of "July Alberta" peach.

Fruit quality

physical fruit

Fruit dimensions proerties and shape

Data presented in Tables 6 and 7 revealed that, raising fruit thinning space and increasing the severity of thinning out and heading back pruning significantly increased both fruit length and diameter.

The interaction was significant in both seasons and the highest values belonged to (To2 x HB2 x FT2), (To2 x HB3 x FT2), (To3 x HB2 x FT2) and (To3 x HB3 x FT2) treatments without significant differences among them and the difference between each of them and the control was significant in both seasons. These results agree with those of Mohsen (2010) on "Florida Prince" and Bussi et al. (2009) on peach and Said et al. (2003) on apricot. Furthermore, Zayan (1991), Siham et al. (2005), and Mikhael (2001) on persimmon.

\section{Fruit shape}

The date of Table 6 and 7 also indicated that fruit shape (L/D ration) was in effected with thinning out and heading back pruning as well as fruit thinning and their interaction in both season. Similar results wear also obtained by Mikhael (2001) .

Average fruit weight and volume $\left(\mathrm{cm}^{3}\right)$

Data in Tables 8 and 9 show that raising fruit spacing at $15 \mathrm{~cm}$ increased fruit weight and volume than those s paced at $10 \mathrm{~cm}$ apart in both seasons. The data also clarify significant increase in average fruit weight by increasing the severity of thinning and heading back treatments and the heaviest fruits were always belonged to severity degree (To3 or HB3). Similar results were obtained by Njorog and Reighard (2008), Zayan (1991) on "Mit Ghamr" peach cv. and Mahajan and Dhillon (2002) on "Sham I Punjab" and Bussi et al. (2009) on "Big Top" and "Alexandra" and Mikhael et al. (2012) on Desert Red peach cv, they found that with increasing the severity of pruning, average fruit weight and volume were significantly increased. However, the hehaviest fruit produced by (To 2 x HB2 x FT2), (To 2 x HB3 x FT2), (To3 x HB2 x FT2) and (To3 x HB3 x FT2) combination treatments, while the lightest fruit obtained by the control(To1 x HB1 x FT1) in both seasons. The difference between wide and narrow fruit spacing was significant in both seasons and the larger fruits were produced by wider fruit spacing at $15 \mathrm{~cm}$. These results herein are in line with those obtained by Mahajan and Dhillon (2002) and Mikhael et al. (2012) mentioned that, fruit volume of "Desert Red" peach significantly increased by increasing the severity of thinning out pruning at dormancy. 
TABLE 6. Effect of thinningout, heading back, fruit thinning and their interaction on dimension and shape index of "Florida Prince" peach fruits in 2009 season.

\begin{tabular}{|c|c|c|c|c|c|c|c|}
\hline \multicolumn{2}{|c|}{ Treatments } & \multicolumn{2}{|c|}{$\begin{array}{c}\text { Fruit length, "L" } \\
(\mathrm{cm})\end{array}$} & \multicolumn{2}{|c|}{$\begin{array}{l}\text { Fruit diameter, } \\
\text { "D" }(\mathrm{cm})\end{array}$} & \multicolumn{2}{|c|}{$\begin{array}{c}\text { Fruit shape L/D } \\
\text { ratio }\end{array}$} \\
\hline $\begin{array}{c}\text { Thinning } \\
\text { out } \\
\text { (To) }\end{array}$ & $\begin{array}{c}\text { Heading back } \\
\text { (HB) }\end{array}$ & \begin{tabular}{|c|}
$\begin{array}{c}\text { Fruit } \\
\text { thinning }\end{array}$ \\
$10 \mathrm{~cm}$ \\
(FT1)
\end{tabular} & \begin{tabular}{|c|}
$\begin{array}{c}\text { Fruit } \\
\text { thinning } \\
15 \mathrm{~cm} \\
\text { (FT2) }\end{array}$
\end{tabular} & $\begin{array}{c}\begin{array}{c}\text { Fruit } \\
\text { thinning } \\
10 \mathrm{~cm}\end{array} \\
\text { (FT1) }\end{array}$ & $\begin{array}{c}\text { Fruit } \\
\text { thinning } \\
15 \mathrm{~cm} \\
\text { (FT2) }\end{array}$ & $\begin{array}{c}\begin{array}{c}\text { Fruit } \\
\text { thinning } \\
10 \mathrm{~cm}\end{array} \\
\text { (FT1) }\end{array}$ & \begin{tabular}{|c}
$\begin{array}{c}\text { Fruit } \\
\text { thinning } \\
15 \mathrm{~cm} \\
\text { (FT2) }\end{array}$ \\
\end{tabular} \\
\hline \multirow{3}{*}{ To1 } & HB1 & 5.20 & 5.59 & 5.42 & 5.82 & 0.96 & 0.96 \\
\hline & HB2 & 5.31 & 5.79 & 5.53 & 6.03 & 0.96 & 0.96 \\
\hline & HB3 & 5.33 & 5.81 & 5.61 & 6.12 & 0.95 & 0.95 \\
\hline \multirow{3}{*}{ To2 } & HB1 & 5.45 & 5.78 & 5.74 & 6.15 & 0.95 & 0.94 \\
\hline & HB2 & 5.81 & 6.05 & 6.12 & 6.51 & 0.95 & 0.93 \\
\hline & HB3 & 5.86 & 6.06 & 6.23 & 6.52 & 0.94 & 0.94 \\
\hline \multirow{3}{*}{ To3 } & HB1 & 5.74 & 5.95 & 6.04 & 6.26 & 0.95 & 0.95 \\
\hline & HB2 & 5.87 & 6.07 & 6.24 & 6.53 & 0.94 & 0.93 \\
\hline & HB3 & 5.91 & 6.09 & 6.35 & 6.55 & 0.94 & 0.93 \\
\hline \multicolumn{2}{|c|}{ L.S.D. interaction 0.05} & \multicolumn{2}{|c|}{0.174} & \multicolumn{2}{|c|}{0.287} & \multicolumn{2}{|c|}{ NS } \\
\hline \multicolumn{2}{|c|}{$\begin{array}{r}0.01 \\
\end{array}$} & \multicolumn{2}{|c|}{0.234} & \multicolumn{2}{|c|}{0.386} & \multicolumn{2}{|c|}{ NS } \\
\hline \multirow{3}{*}{$\begin{array}{l}\text { Mean } \\
\text { effect of } \\
\text { thinning } \\
\text { out }\end{array}$} & $\begin{array}{c}\text { Thinning out } \\
25 \%\end{array}$ & \multicolumn{2}{|c|}{5.51} & \multicolumn{2}{|c|}{5.76} & \multicolumn{2}{|c|}{0.96} \\
\hline & $\begin{array}{c}\text { Thinning out } \\
50 \% \\
\end{array}$ & \multicolumn{2}{|c|}{5.84} & \multicolumn{2}{|c|}{6.21} & \multicolumn{2}{|c|}{0.94} \\
\hline & $\begin{array}{c}\text { Thinning out } \\
75 \% \\
\end{array}$ & \multicolumn{2}{|c|}{5.94} & \multicolumn{2}{|c|}{6.33} & \multicolumn{2}{|c|}{0.94} \\
\hline \multicolumn{2}{|c|}{ L.S.D. 0.05} & \multicolumn{2}{|c|}{0.071} & \multicolumn{2}{|c|}{0.117} & \multicolumn{2}{|c|}{ NS } \\
\hline \multicolumn{2}{|c|}{ L.S.D. 0.01} & \multicolumn{2}{|c|}{0.095} & \multicolumn{2}{|c|}{0.158} & $\mathrm{~N}$ & JS \\
\hline Mean & Heading back $0 \%$ & & 62 & 5. & 91 & 0. & 95 \\
\hline $\begin{array}{l}\text { effect of } \\
\text { heading }\end{array}$ & $\begin{array}{c}\text { Heading back } \\
25 \% \\
\end{array}$ & & 82 & & 16 & 0 . & 95 \\
\hline back & $\begin{array}{c}\text { Heading back } \\
50 \%\end{array}$ & & 84 & 6. & 23 & 0. & 94 \\
\hline & S.D. 0.05 & & 071 & 0. & 117 & $\mathrm{~N}$ & $\mathrm{JS}$ \\
\hline & S.D. 0.01 & & 095 & 0. & 158 & $\mathrm{~N}$ & JS \\
\hline $\begin{array}{c}\text { Mean } \\
\text { effect of }\end{array}$ & \begin{tabular}{|c|}
$\begin{array}{c}\text { Fruit thinning } 10 \\
\mathrm{~cm}\end{array}$ \\
\end{tabular} & & 61 & 5 . & 92 & 0 . & 95 \\
\hline $\begin{array}{c}\text { fruit } \\
\text { thinning }\end{array}$ & $\begin{array}{c}\begin{array}{c}\text { Fruit thinning } 15 \\
\mathrm{~cm}\end{array} \\
\end{array}$ & & 91 & 6. & 28 & 0 . & 94 \\
\hline & S.D. 0.05 & & 058 & 0. & 096 & $\mathrm{~N}$ & JS \\
\hline & S.D. 0.01 & & 078 & 0. & 129 & $\mathrm{~N}$ & JS \\
\hline
\end{tabular}

Egypt. J. Hort. Vol. 42, No.1 (2015) 
IMPROVING PRODUCTIVITY AND FRUIT QUALITY OF FLORIDA ...

TABLE 7. Effect of thinning out, heading back, fruit thinning and their interaction on dimension and shape index of "Florida Prince" peach fruits in 2010 season.

\begin{tabular}{|c|c|c|c|c|c|c|c|}
\hline \multicolumn{2}{|c|}{ Treatments } & \multicolumn{2}{|c|}{$\begin{array}{l}\text { Fruit length, "L" } \\
(\mathbf{c m})\end{array}$} & \multicolumn{2}{|c|}{$\begin{array}{l}\text { Fruit diameter, } \\
\text { "D" }(\mathrm{cm})\end{array}$} & \multicolumn{2}{|c|}{$\begin{array}{c}\text { Fruit shape L/D } \\
\text { ratio }\end{array}$} \\
\hline $\begin{array}{c}\text { Thinning } \\
\text { out } \\
\text { (To) }\end{array}$ & $\begin{array}{c}\text { Heading back } \\
\text { (HB) }\end{array}$ & 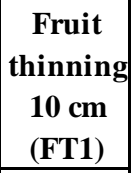 & $\begin{array}{c}\begin{array}{c}\text { Fruit } \\
\text { thinning } \\
15 \mathrm{~cm} \\
(\text { FT2) }\end{array} \\
\end{array}$ & 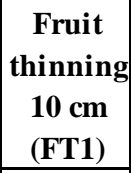 & \begin{tabular}{|c}
$\begin{array}{c}\text { Fruit } \\
\text { thinning } \\
15 \mathrm{~cm} \\
\text { (FT2) }\end{array}$ \\
\end{tabular} & $\begin{array}{c}\begin{array}{c}\text { Fruit } \\
\text { thinning } \\
10 \mathrm{~cm} \\
\text { (FT1) }\end{array} \\
\end{array}$ & $\begin{array}{c}\begin{array}{c}\text { Fruit } \\
\text { thinning } \\
15 \mathrm{~cm} \\
\text { (FT2) }\end{array} \\
\end{array}$ \\
\hline \multirow{3}{*}{ Tol } & HB1 & 5.30 & \begin{tabular}{|l|}
5.65 \\
\end{tabular} & 5.46 & 5.89 & 0.97 & 0.96 \\
\hline & HB2 & 5.36 & 5.86 & 5.58 & 6.10 & 0.96 & 0.96 \\
\hline & HB3 & 5.41 & 5.85 & 5.64 & 6.16 & 0.96 & 0.95 \\
\hline \multirow{3}{*}{ To2 } & HB1 & 5.53 & 5.96 & 5.76 & 6.21 & 0.96 & 0.96 \\
\hline & HB2 & 5.87 & 6.17 & 6.18 & 6.56 & 0.95 & 0.94 \\
\hline & HB3 & 6.07 & 6.12 & 6.39 & 6.58 & 0.95 & 0.93 \\
\hline \multirow{3}{*}{ To3 } & HB1 & 5.79 & 5.95 & 6.09 & 6.31 & 0.95 & 0.95 \\
\hline & HB2 & 5.94 & 6.18 & 6.36 & 6.57 & 0.4 & 0.94 \\
\hline & HB3 & 6.04 & 6.12 & 6.49 & 6.58 & 0.93 & 0.93 \\
\hline \multicolumn{2}{|c|}{ L.S.D. interaction 0.05} & \multicolumn{2}{|c|}{0.174} & \multicolumn{2}{|c|}{0.189} & \multicolumn{2}{|c|}{ NS } \\
\hline \multicolumn{2}{|r|}{0.01} & \multicolumn{2}{|c|}{0.234} & \multicolumn{2}{|c|}{0.254} & \multicolumn{2}{|c|}{ NS } \\
\hline \multirow{3}{*}{$\begin{array}{c}\text { Mean } \\
\text { effect of } \\
\text { thinning } \\
\text { out }\end{array}$} & $\begin{array}{c}\text { Thinning out } \\
25 \%\end{array}$ & \multicolumn{2}{|c|}{5.57} & \multicolumn{2}{|c|}{5.81} & \multicolumn{2}{|c|}{0.96} \\
\hline & $\begin{array}{c}\text { Thinning out } \\
50 \%\end{array}$ & \multicolumn{2}{|c|}{5.95} & \multicolumn{2}{|c|}{6.28} & \multicolumn{2}{|c|}{0.95} \\
\hline & $\begin{array}{c}\text { Thinning out } \\
75 \%\end{array}$ & \multicolumn{2}{|c|}{6.01} & \multicolumn{2}{|c|}{6.40} & \multicolumn{2}{|c|}{0.94} \\
\hline \multicolumn{2}{|c|}{ L.S.D. 0.05} & \multicolumn{2}{|c|}{0.071} & \multicolumn{2}{|c|}{0.077} & \multicolumn{2}{|c|}{ NS } \\
\hline \multicolumn{2}{|c|}{ L.S.D. 0.01} & \multicolumn{2}{|c|}{0.095} & \multicolumn{2}{|c|}{0.104} & $\mathrm{~N}$ & $\mathrm{JS}$ \\
\hline $\begin{array}{c}\text { Mean } \\
\text { effect of }\end{array}$ & $\begin{array}{c}\text { Heading back } \\
0 \%\end{array}$ & 5 . & 70 & 5. & 95 & 0 . & 96 \\
\hline $\begin{array}{l}\text { heading } \\
\text { back }\end{array}$ & $\begin{array}{c}\text { Heading back } \\
25 \%\end{array}$ & 5. & 91 & 6. & 23 & 0. & 95 \\
\hline & $\begin{array}{c}\text { Heading back } \\
50 \% \\
\end{array}$ & 9. & 94 & 6. & 31 & 0 . & 94 \\
\hline L.S & S.D. 0.05 & 0.0 & 71 & 0.0 & 977 & $\mathrm{~N}$ & IS \\
\hline & S.D. 0.01 & 0.0 & 995 & 0.1 & 104 & & IS \\
\hline $\begin{array}{c}\text { Mean } \\
\text { effect of }\end{array}$ & $\begin{array}{c}\text { Fruit thinning } \\
10 \mathrm{~cm}\end{array}$ & 5. & 71 & 5. & 99 & 0 . & 95 \\
\hline $\begin{array}{c}\text { fruit } \\
\text { thinning }\end{array}$ & $\begin{array}{l}\text { Fruit thinning } \\
\qquad 15 \mathrm{~cm}\end{array}$ & 5. & 98 & 6. & 33 & 0. & 95 \\
\hline L.S & S.D. 0.05 & 0.0 & 58 & 0.0 & 63 & $\mathrm{~N}$ & JS \\
\hline L.S & D.D. 0.01 & 0.0 & 978 & 0.0 & 885 & & IS \\
\hline
\end{tabular}


TABLE 8 Effect of thinning out, heading back, fruit thinning and their interaction on some physical properties of "Florida Prince" peach fruits in 2009 season.

\begin{tabular}{|c|c|c|c|c|c|c|c|}
\hline \multicolumn{2}{|c|}{ Treatments } & \multicolumn{2}{|c|}{ Av. fruit weight (g) } & \multicolumn{2}{|c|}{$\begin{array}{l}\text { Av. fruit volume } \\
\left(\mathrm{cm}^{2}\right)\end{array}$} & \multicolumn{2}{|c|}{ Firmness (Lb/inch ${ }^{2}$} \\
\hline $\begin{array}{c}\text { Thinning } \\
\text { out } \\
\text { (To) }\end{array}$ & $\begin{array}{l}\text { Heading back } \\
\text { (HB) }\end{array}$ & $\begin{array}{c}\begin{array}{c}\text { Fruit } \\
\text { thinning } \\
10 \mathrm{~cm}\end{array} \\
\text { (FT1) } \\
\end{array}$ & $\begin{array}{c}\text { Fruit } \\
\text { thinning } \\
15 \mathrm{~cm} \\
\text { (FT2) }\end{array}$ & 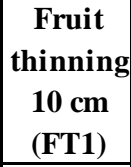 & $\begin{array}{c}\begin{array}{c}\text { Fruit } \\
\text { thinning }\end{array} \\
15 \mathrm{~cm} \\
\text { (FT2) } \\
\end{array}$ & $\begin{array}{c}\text { Fruit } \\
\text { thinning } \\
10 \mathrm{~cm} \\
\text { (FT1) }\end{array}$ & 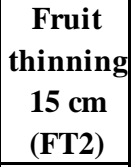 \\
\hline \multirow{3}{*}{ To1 } & HB1 & 78.12 & 91.45 & 76.17 & 89.15 & 11.84 & 11.12 \\
\hline & HB2 & 87.76 & 103.74 & 86.38 & 101.04 & 11.28 & 10.10 \\
\hline & HB3 & 91.88 & 107.06 & 88.94 & 105.03 & 10.85 & 9.26 \\
\hline \multirow{3}{*}{ To2 } & HB1 & 85.14 & 94.53 & 82.42 & 91.88 & 11.56 & 9.77 \\
\hline & HB2 & 93.71 & 117.82 & 90.61 & 115.37 & 11.49 & 8.89 \\
\hline & HB3 & 97.96 & 118.91 & 95.52 & 115.86 & 10.36 & 8.64 \\
\hline \multirow{3}{*}{ To3 } & HB1 & 87.71 & 106.98 & 85.43 & 104.63 & 10.61 & 9.35 \\
\hline & HB2 & 95.83 & 116.45 & 93.24 & 113.89 & 9.72 & 8.69 \\
\hline & HB3 & 101.34 & 118.28 & 98.09 & 115.85 & 9.58 & 8.42 \\
\hline \multicolumn{2}{|c|}{ L.S.D. interaction 0.05} & \multicolumn{2}{|c|}{6.996} & \multicolumn{2}{|c|}{7.051} & \multicolumn{2}{|c|}{0.222} \\
\hline \multicolumn{2}{|c|}{0.01} & \multicolumn{2}{|c|}{9.355} & \multicolumn{2}{|c|}{9.482} & \multicolumn{2}{|c|}{0.299} \\
\hline \multirow{3}{*}{$\begin{array}{l}\text { Mean } \\
\text { effect of } \\
\text { thinning } \\
\text { out }\end{array}$} & $\begin{array}{c}\text { Thinning out } \\
25 \% \\
\end{array}$ & \multicolumn{2}{|c|}{93.34} & \multicolumn{2}{|c|}{91.12} & \multicolumn{2}{|c|}{10.74} \\
\hline & $\begin{array}{c}\text { Thinning out } \\
50 \% \\
\end{array}$ & \multicolumn{2}{|c|}{101.35} & \multicolumn{2}{|c|}{98.61} & \multicolumn{2}{|c|}{10.12} \\
\hline & $\begin{array}{c}\text { Thinning out } \\
75 \% \\
\end{array}$ & \multicolumn{2}{|c|}{104.43} & \multicolumn{2}{|c|}{101.86} & \multicolumn{2}{|c|}{9.40} \\
\hline \multicolumn{2}{|c|}{ L.S.D. 0.05} & \multicolumn{2}{|c|}{2.840} & \multicolumn{2}{|c|}{2.878} & \multicolumn{2}{|c|}{0.091} \\
\hline \multicolumn{2}{|c|}{ L.S.D. 0.01} & \multicolumn{2}{|c|}{3.819} & \multicolumn{2}{|c|}{3.871} & 0.1 & 22 \\
\hline $\begin{array}{l}\text { Mean } \\
\text { effect of }\end{array}$ & $\begin{array}{c}\text { Heading back } \\
0 \% \\
\end{array}$ & 90 & .66 & 88 & 28 & & .71 \\
\hline $\begin{array}{c}\text { heading } \\
\text { back }\end{array}$ & $\begin{array}{c}\text { Heading back } \\
25 \% \\
\end{array}$ & 102 & 2.55 & 100 & .09 & & .03 \\
\hline & $\begin{array}{c}\text { Heading back } \\
50 \% \\
\end{array}$ & 10 & 5.91 & 103 & .22 & & 52 \\
\hline L.S & S.D. 0.05 & 2.8 & 840 & 2.8 & 378 & & 91 \\
\hline L.S & S.D. 0.01 & 3.8 & 819 & 3.8 & 371 & & 22 \\
\hline $\begin{array}{c}\text { Mean } \\
\text { effect of }\end{array}$ & $\begin{array}{c}\text { Fruit thinning } 10 \\
\mathrm{~cm}\end{array}$ & 91 & .05 & 88 & .53 & & 81 \\
\hline $\begin{array}{c}\text { fruit } \\
\text { thinning }\end{array}$ & $\begin{array}{c}\text { Fruit thinning } 15 \\
\mathrm{~cm}\end{array}$ & 108 & 8.36 & 105 & .86 & & 36 \\
\hline L.S & S.D. 0.05 & 2.3 & 318 & 2.3 & 50 & & 74 \\
\hline L.S & S.D. 0.01 & 3.1 & 118 & 3.1 & 60 & & 00 \\
\hline
\end{tabular}

Egypt. J. Hort. Vol. 42, No.1 (2015) 
TABLE 9. Effect of thinning out, heading back, fruit thinning and their interaction on some physical properties of "Florida Prince" peach fruits in 2010 season.

\begin{tabular}{|c|c|c|c|c|c|c|c|}
\hline \multicolumn{2}{|c|}{ Treatments } & \multicolumn{2}{|c|}{ Av. fruit weight (g) } & \multicolumn{2}{|c|}{$\begin{array}{c}\text { Av. fruit volume } \\
\left(\mathrm{cm}^{2}\right)\end{array}$} & \multicolumn{2}{|c|}{ Firmness (Lb/inch ${ }^{2}$} \\
\hline $\begin{array}{c}\text { Thinning } \\
\text { out } \\
\text { (To) }\end{array}$ & $\begin{array}{c}\text { Heading back } \\
\text { (HB) }\end{array}$ & 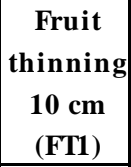 & 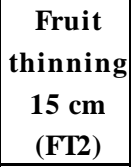 & \begin{tabular}{|c|}
$\begin{array}{c}\text { Fruit } \\
\text { thinning } \\
10 \mathrm{~cm} \\
(\text { FT1 })\end{array}$ \\
\end{tabular} & $\begin{array}{c}\text { Fruit } \\
\text { thinning } \\
15 \mathrm{~cm} \\
\text { (FT2) }\end{array}$ & $\begin{array}{c}\text { Fruit } \\
\text { thinning } \\
10 \mathrm{~cm} \\
\text { (FT1) }\end{array}$ & $\begin{array}{c}\begin{array}{c}\text { Fruit } \\
\text { thinning } \\
15 \mathrm{~cm} \\
\text { (FT2) }\end{array} \\
\end{array}$ \\
\hline \multirow{3}{*}{ To1 } & HB1 & 82.36 & 95.40 & 80.95 & 93.69 & 11.65 & 10.92 \\
\hline & HB2 & 88.43 & 107.26 & 85.72 & 104.83 & 11.16 & 9.96 \\
\hline & HB3 & 94.62 & 109.42 & 92.07 & 106.78 & 10.65 & 8.92 \\
\hline \multirow{3}{*}{ To2 } & HB1 & 88.29 & 97.85 & 86.61 & 96.19 & 11.34 & 9.59 \\
\hline & HB2 & 96.82 & 120.74 & 95.46 & 118.81 & 10.28 & 8.72 \\
\hline & HB3 & 100.77 & 120.92 & 97.95 & 118.98 & 10.19 & 8.54 \\
\hline \multirow{3}{*}{ To3 } & HB1 & 91.28 & 108.55 & 88.54 & 105.51 & 10.42 & 9.18 \\
\hline & HB2 & 98.57 & 118.62 & 95.71 & 116.25 & 9.61 & 8.71 \\
\hline & HB3 & 105.11 & 120.57 & 103.75 & 117.64 & 9.36 & 8.18 \\
\hline \multicolumn{2}{|c|}{ L.S.D. interaction 0.05} & \multicolumn{2}{|c|}{5.953} & \multicolumn{2}{|c|}{6.320} & \multicolumn{2}{|c|}{0.203} \\
\hline \multicolumn{2}{|c|}{0.01} & \multicolumn{2}{|c|}{8.006} & \multicolumn{2}{|c|}{8.486} & \multicolumn{2}{|c|}{0.273} \\
\hline \multirow{3}{*}{$\begin{array}{c}\text { Mean } \\
\text { effect of } \\
\text { thinning } \\
\text { out }\end{array}$} & $\begin{array}{c}\text { Thinning out } \\
25 \% \\
\end{array}$ & \multicolumn{2}{|c|}{96.25} & \multicolumn{2}{|c|}{94.01} & \multicolumn{2}{|c|}{10.54} \\
\hline & $\begin{array}{c}\text { Thinning out } \\
50 \% \\
\end{array}$ & \multicolumn{2}{|c|}{104.23} & \multicolumn{2}{|c|}{102.33} & \multicolumn{2}{|c|}{9.78} \\
\hline & $\begin{array}{c}\text { Thinning out } \\
75 \%\end{array}$ & \multicolumn{2}{|c|}{107.12} & \multicolumn{2}{|c|}{104.57} & \multicolumn{2}{|c|}{9.24} \\
\hline \multicolumn{2}{|c|}{ L.S.D. 0.05} & \multicolumn{2}{|c|}{2.430} & \multicolumn{2}{|c|}{2.580} & \multicolumn{2}{|c|}{0.083} \\
\hline \multicolumn{2}{|c|}{ L.S.D. 0.01} & \multicolumn{2}{|c|}{3.268} & \multicolumn{2}{|c|}{3.464} & 0.1 & 114 \\
\hline $\begin{array}{c}\text { Mean } \\
\text { effect of }\end{array}$ & $\begin{array}{c}\text { Heading back } \\
0 \% \\
\end{array}$ & 93 & 96 & & .92 & 10 & .52 \\
\hline $\begin{array}{l}\text { heading } \\
\text { back }\end{array}$ & $\begin{array}{c}\text { Heading back } \\
25 \% \\
\end{array}$ & 105 & 5.07 & 102 & 2.80 & 9. & 74 \\
\hline & $\begin{array}{c}\text { Heading back } \\
50 \%\end{array}$ & 107 & 7.57 & 106 & 6.20 & 9. & 31 \\
\hline & S.D. 0.05 & 2.4 & 130 & 2.5 & 580 & 0.0 & 83 \\
\hline & S.D. 0.01 & 3.2 & 268 & & 464 & & 114 \\
\hline $\begin{array}{c}\text { Mean } \\
\text { effect of }\end{array}$ & \begin{tabular}{|c|}
$\begin{array}{c}\text { Fruit thinning } 10 \\
\mathrm{~cm}\end{array}$ \\
\end{tabular} & 94 & .03 & & .86 & 10 & .52 \\
\hline $\begin{array}{c}\text { fruit } \\
\text { thinning }\end{array}$ & $\begin{array}{c}\text { Fruit thinning } 15 \\
\mathrm{~cm}\end{array}$ & 111 & 1.04 & 108 & 8.74 & 9. & 19 \\
\hline & S.D. 0.05 & 1.9 & 84 & & 186 & & 68 \\
\hline & S.D. 0.01 & 2.6 & 669 & & 940 & & 991 \\
\hline
\end{tabular}


Fruit firmness ( Lb/inch $\left.^{2}\right)$

Data presented in Tables 8 and 9, clear that raising thinning out and heading back as well as fruit thinning levels led to decrease fruit firmness in both seasons. These reduction in fruit firmness might be due to the increase of fruit size and the reduction in its $\mathrm{Ca}$ concentration. These findings confirmed with those obtained by Stino (1995)and Demitras et al. (2010), Samara et al. (2003) and Mohsen (2010) indicated that hand fruit thinning significantly reduced fruit firmness. On the other hand, Attala (1997) and Njorog and Reighard (2008) showed that fruit thinning did not influence fruit firmness. However, the interaction (To x HB x FT) was significant and the firm fruits came from light thinning out and un-headed trees with narrow fruit spacing in (To1 xHb1 xFT1) treatment.

\section{Chemical fruit properties}

Data in Tables 10-11 show that TSS value and TSS/acid ratio were significantly increased by increasing the severity of thinning out and heading back pruning. The interaction was significant in both seasons and the highest values achieved by (TO2 x HB2 x FT2) compared to the least values obtained by TO1 x HB1 x FT1. On the other hand, the same treatment and the interaction reduced the acidity in both seasons. These results are supported by conclusion of Zayan (1991), Mikhael et al. (2012) on peach cvs.

Concerning vit. $\mathrm{C}$ content in the same tables data clear that all the tested thinned out and heading back pruning treatments significantly increased vit. C. Fruit spaced at $15 \mathrm{~cm}$ with higher vit. C. These results are in agreement with the findings of Attala (1997) and Abo Ogiela (2006).

\section{Fruit colour}

Data presented in Table 12 show that, the degree of red colour and the values of Ancocyanin content in each fruit skin were increased by increasing the degree of both thinning out up to 50 or $75 \%$ and heading back up to 25 or $50 \%$ and increasing the spacing between fruit from 10 to $15 \mathrm{~cm}$ apart. The increment was significant in both seasons. the abovementioned results are in accordance with those reported by Zayan et al. (2002), Mika (1986) and Samara et al. (2003) which they mentioned that hand thinning increased ancocyanin content in "Anna" apple fruit compared to un thinned trees.

Finally, it can be recommended Thinning out $50 \%$ and heading back $25 \%$ of one year old shoots with fruit Thinning at $15 \mathrm{~cm}$ a part obtain the highest yield with highly physical and chemical fruit characters.

Egypt. J. Hort. Vol. 42, No.1 (2015) 
IMPROVING PRODUCTIVITY AND FRUIT QUALITY OF FLORIDA ...

TABLE 10. Effect of thinning out, heading back, fruit thinning and their interaction on some chemical properties of "Florida Prince" peach fruits in 2009 season.

\begin{tabular}{|c|c|c|c|c|c|c|c|c|c|}
\hline \multicolumn{2}{|c|}{ Treatment } & \multicolumn{2}{|c|}{ TSS \% } & \multicolumn{2}{|c|}{ Acidity \% } & \multicolumn{2}{|c|}{ TSS/acidity ratio } & \multicolumn{2}{|c|}{$\begin{array}{c}\text { Vit C. mg/100 } \\
\text { g/fruit }\end{array}$} \\
\hline \multirow[t]{2}{*}{$\begin{array}{c}\text { Thinning } \\
\text { out (To) }\end{array}$} & \multirow[t]{2}{*}{$\begin{array}{c}\text { Heading back } \\
\text { (HB) }\end{array}$} & \multirow{2}{*}{$\begin{array}{c}\text { Fruit } \\
\text { thinning } \\
10 \mathrm{~cm} \\
\text { (FT1) }\end{array}$} & \multirow{2}{*}{$\begin{array}{l}\text { Fruit } \\
\text { thinning } \\
15 \mathrm{~cm} \\
\text { (FT2) }\end{array}$} & \multirow{2}{*}{$\begin{array}{c}\text { Fruit } \\
\text { thinning } \\
10 \mathrm{~cm} \\
(\mathrm{FT1})\end{array}$} & \multirow{2}{*}{$\begin{array}{l}\text { Fruit } \\
\text { thinning } \\
15 \mathrm{~cm} \\
\text { (FT2) }\end{array}$} & \multicolumn{2}{|c|}{$\begin{array}{c}\text { Fruit } \text { Fruit } \\
\text { thinningthinnin }\end{array}$} & \multirow{2}{*}{$\begin{array}{l}\text { Fruit } \\
\text { thinnin } \\
10 \mathrm{~cm} \\
\text { (FT1) }\end{array}$} & \multirow{2}{*}{$\begin{array}{l}\text { Fruit } \\
\text { thinning } \\
15 \mathrm{~cm} \\
\text { (FT2) }\end{array}$} \\
\hline & & & & & & $\begin{array}{l}10 \mathrm{~cm} \\
(\text { FT1 })\end{array}$ & $\begin{array}{r}15 \mathrm{~cm} \\
(\mathrm{FT} 2)\end{array}$ & & \\
\hline \multirow{3}{*}{ To1 } & HB1 & 9.27 & 10.47 & 1.04 & 0.95 & 9.46 & 9.56 & 8.91 & 11.02 \\
\hline & HB2 & 9.53 & 10.87 & 0.98 & 0.92 & 10.33 & 11.42 & 9.72 & 11.82 \\
\hline & HB3 & 9.67 & 11.13 & 0.95 & 0.91 & 11.86 & 12.03 & 10.18 & 12.23 \\
\hline \multirow{3}{*}{ To2 } & HB1 & 10.40 & 10.80 & 0.97 & 0.86 & 9.69 & 9.66 & 10.72 & 12.56 \\
\hline & HB2 & 10.80 & 11.87 & 0.91 & 0.82 & 11.46 & 12.83 & 11.87 & 14.48 \\
\hline & HB3 & 11.13 & 11.80 & 0.88 & 0.82 & 12.06 & 12.53 & 12.65 & 14.39 \\
\hline \multirow{3}{*}{ To3 } & HB1 & 10.73 & 11.40 & 0.93 & 0.85 & 9.43 & 9.67 & 11.54 & 13.41 \\
\hline & HB2 & 11.20 & 11.93 & 0.86 & 0.82 & 11.56 & 12.13 & 13.02 & 14.55 \\
\hline & HB3 & 11.30 & 12.00 & 0.84 & 0.80 & 11.66 & 12.20 & 13.81 & 15.00 \\
\hline \multicolumn{2}{|c|}{ L.S.D. interaction 0.05} & \multicolumn{2}{|c|}{0.356} & \multicolumn{2}{|c|}{0.052} & \multicolumn{2}{|c|}{0.501} & \multicolumn{2}{|c|}{0.778} \\
\hline \multicolumn{2}{|c|}{0.01} & \multicolumn{2}{|c|}{0.478} & \multicolumn{2}{|c|}{0.071} & \multicolumn{2}{|c|}{0.672} & \multicolumn{2}{|c|}{1.045} \\
\hline \multirow{3}{*}{\begin{tabular}{|c|} 
Mean effect \\
of thinning \\
out
\end{tabular}} & Thinning out $25 \%$ & \multicolumn{2}{|c|}{10.16} & \multicolumn{2}{|c|}{0.96} & 10 & 78 & 10 & 65 \\
\hline & Thinning out $50 \%$ & 11 & & 0. & 88 & 11 & 37 & & .78 \\
\hline & Thinning out $75 \%$ & 11. & & 0. & 85 & 11 & 11 & & 56 \\
\hline & S.D. 0.05 & 0.1 & & 0.0 & 21 & 0.2 & 04 & 0.3 & 18 \\
\hline & S.D. 0.01 & 0.1 & & 0.0 & 29 & 0.2 & 74 & 0.4 & 27 \\
\hline Mean effect & Heading back $0 \%$ & 10 & & 0. & 93 & 9. & 58 & 11 & 36 \\
\hline of heading & Heading back $25 \%$ & 11 & & 0. & 89 & 11 & 62 & & .58 \\
\hline & Heading back 50\% & 11 & 22 & 0. & 87 & 12 & 06 & & .04 \\
\hline L.S & S.D. 0.05 & 0.1 & 45 & 0.0 & 21 & 0.2 & 04 & & 18 \\
\hline L.S & S.D. 0.01 & 0.1 & 95 & 0.0 & 29 & 0.2 & 74 & & 27 \\
\hline $\begin{array}{c}\text { Mean effect } \\
\text { of fruit }\end{array}$ & $\begin{array}{c}\text { Fruit thinning 10 } \\
\mathrm{cm}\end{array}$ & 10 & 48 & 0. & 93 & 10 & 83 & & 38 \\
\hline thinning & $\begin{array}{c}\text { Fruit thinning } 15 \\
\mathrm{~cm}\end{array}$ & 11 & 36 & 0. & 86 & 11 & 34 & & 27 \\
\hline L.S & S.D. 0.05 & 0.1 & 19 & 0.0 & 17 & 0.1 & 67 & & 59 \\
\hline L.S & S.D. 0.01 & 0.1 & 60 & 0.0 & 24 & 0.0 & 24 & & 49 \\
\hline
\end{tabular}

Egypt. J. Hort. Vol. 42, No.1 (2015) 
TABLE 11. Effect of thinning out, heading back, fruit thinning and their interaction on some chemical properties of "Florida Prince" peach fruits in 2010 season.

\begin{tabular}{|c|c|c|c|c|c|c|c|c|c|}
\hline \multicolumn{2}{|c|}{ Treatment } & \multicolumn{2}{|c|}{ TSS \% } & \multicolumn{2}{|c|}{ Acidity \% } & \multicolumn{2}{|c|}{ TSS/acidity ratio } & \multicolumn{2}{|c|}{$\begin{array}{l}\text { Vit C. mg/100 } \\
\text { g/fruit }\end{array}$} \\
\hline $\begin{array}{l}\text { Thinning } \\
\text { out (To) }\end{array}$ & $\begin{array}{c}\text { Heading } \\
\text { back }\end{array}$ & $\begin{array}{c}\text { Fruit } \\
\text { thinning }\end{array}$ & \begin{tabular}{|c|} 
Fruit \\
thinning
\end{tabular} & \begin{tabular}{|c|} 
Fruit \\
thinning
\end{tabular} & $\begin{array}{l}\text { Fruit } \\
\text { thinning }\end{array}$ & \begin{tabular}{|c|} 
Fruit \\
thinning
\end{tabular} & \begin{tabular}{|c|} 
Fruit \\
thinning
\end{tabular} & \begin{tabular}{|c|} 
Fruit \\
thinning
\end{tabular} & $\begin{array}{c}\text { Fruit } \\
\text { thinning }\end{array}$ \\
\hline & $(\mathbf{H B})$ & $\begin{array}{l}10 \mathrm{~cm} \\
\text { (FT1) }\end{array}$ & $\begin{array}{l}15 \mathrm{~cm} \\
\text { (FT2) }\end{array}$ & $\begin{array}{l}10 \mathrm{~cm} \\
\text { (FT1) }\end{array}$ & $\begin{array}{l}15 \mathrm{~cm} \\
\text { (FT2) }\end{array}$ & $\begin{array}{l}10 \mathrm{~cm} \\
\text { (FT1) }\end{array}$ & $\begin{array}{l}15 \mathrm{~cm} \\
\text { (FT2) }\end{array}$ & $\begin{array}{l}10 \mathrm{~cm} \\
\text { (FT1) }\end{array}$ & $\begin{array}{l}15 \mathrm{~cm} \\
\text { (FT2) }\end{array}$ \\
\hline \multirow{3}{*}{ Tol } & HB1 & 9.20 & 10.40 & 1.01 & 0.94 & 9.40 & 9.46 & 9.11 & 11.06 \\
\hline & HB2 & 9.33 & 10.47 & 0.96 & 0.91 & 11.16 & 12.06 & 9.72 & 11.51 \\
\hline & HB3 & 9.53 & 11.07 & 0.93 & 0.90 & 11.23 & 12.20 & 10.25 & 12.30 \\
\hline \multirow{3}{*}{ To2 } & HB1 & 10.27 & 10.67 & 0.95 & 0.85 & 9.66 & 9.70 & 10.81 & 12.55 \\
\hline & HB2 & 10.73 & 11.73 & 0.87 & 0.81 & 11.90 & 13.20 & 12.33 & 14.48 \\
\hline & HB3 & 11.07 & 11.67 & 0.86 & 0.80 & 11.50 & 13.00 & 12.87 & 14.59 \\
\hline \multirow{3}{*}{ To3 } & HB1 & 10.60 & 11.33 & 0.92 & 0.84 & 9.56 & 9.83 & 11.52 & 13.49 \\
\hline & HB2 & 11.27 & 11.80 & 0.85 & 0.81 & 11.23 & 12.50 & 13.26 & 14.57 \\
\hline & HB3 & 11.53 & 11.87 & 0.83 & 0.78 & 11.86 & 12.23 & 13.89 & 15.22 \\
\hline \multicolumn{2}{|c|}{$\begin{array}{l}\text { L.S.D. interaction } \\
0.05\end{array}$} & \multicolumn{2}{|c|}{0.340} & \multicolumn{2}{|c|}{0.054} & \multicolumn{2}{|c|}{0.469} & \multicolumn{2}{|c|}{0.813} \\
\hline \multicolumn{2}{|c|}{0.01} & \multicolumn{2}{|c|}{0.457} & \multicolumn{2}{|c|}{0.073} & \multicolumn{2}{|c|}{0.630} & \multicolumn{2}{|c|}{1.091} \\
\hline \multirow{3}{*}{$\begin{array}{c}\text { Mean } \\
\text { effect of } \\
\text { thinning } \\
\text { out }\end{array}$} & $\begin{array}{l}\text { Thinning } \\
\text { out } 25 \%\end{array}$ & \multicolumn{2}{|c|}{10.00} & \multicolumn{2}{|c|}{0.94} & \multicolumn{2}{|c|}{10.92} & \multicolumn{2}{|c|}{10.66} \\
\hline & $\begin{array}{l}\text { Thinning } \\
\text { out } 50 \%\end{array}$ & \multicolumn{2}{|c|}{11.02} & \multicolumn{2}{|c|}{0.86} & \multicolumn{2}{|c|}{11.49} & \multicolumn{2}{|c|}{12.94} \\
\hline & $\begin{array}{l}\text { Thinning } \\
\text { out } 75 \%\end{array}$ & \multicolumn{2}{|c|}{11.40} & \multicolumn{2}{|c|}{0.84} & \multicolumn{2}{|c|}{11.20} & & .66 \\
\hline L.S.D. & 0.05 & 0.1 & 139 & 0.0 & 22 & 0.1 & 92 & 0.3 & 332 \\
\hline L.S.D. & 0.01 & 0.1 & 186 & 0.0 & 30 & 0.2 & 257 & & 446 \\
\hline $\begin{array}{c}\text { Mean } \\
\text { effect of }\end{array}$ & $\begin{array}{l}\text { Heading } \\
\text { back } 0 \%\end{array}$ & 10 . & .41 & 0. & 92 & 9. & 60 & & .42 \\
\hline $\begin{array}{c}\text { heading } \\
\text { back }\end{array}$ & $\begin{array}{c}\text { Heading } \\
\text { back } \\
25 \%\end{array}$ & 10 . & .89 & 0 . & 87 & 12 . & .01 & & .65 \\
\hline & $\begin{array}{c}\text { Heading } \\
\text { back } \\
50 \%\end{array}$ & 11. & .12 & 0 . & 85 & 12 . & .00 & & .19 \\
\hline L.S.D. & 0.05 & 0.1 & 139 & 0.0 & 22 & 0.1 & 92 & & 332 \\
\hline L.S.D. & 0.01 & 0.1 & 186 & $\overline{0.0}$ & 30 & 0.2 & 257 & & 446 \\
\hline $\begin{array}{c}\text { Mean } \\
\text { effect of } \\
\text { fruit }\end{array}$ & $\begin{array}{c}\text { Fruit } \\
\text { thinning } \\
10 \mathrm{~cm}\end{array}$ & 10 . & .28 & 0 . & 91 & 10 . & .83 & & .53 \\
\hline thinning & $\begin{array}{c}\text { Fruit } \\
\text { thinning } \\
15 \mathrm{~cm}\end{array}$ & 11. & .22 & 0 . & 85 & 11. & .58 & & .31 \\
\hline L.S.D. & 0.05 & 0.1 & 113 & 0.0 & 518 & 0.1 & 56 & & 271 \\
\hline L.S.D. & 0.01 & 0.1 & 152 & 0.0 & 24 & 0.2 & 210 & & 364 \\
\hline
\end{tabular}

Egypt. J. Hort. Vol. 42, No.1 (2015) 
IMPROVING PRODUCTIVITY AND FRUIT QUALITY OF FLORIDA ...

TABLE 12.Effect of thinning out, heading back, fruit thinning and their interaction on colour degree and anthocyanin content of "Florida Prince" peach fruits in 2009 and 2010 seasons $(1=$ green, $10=$ full red).

\begin{tabular}{|c|c|c|c|c|c|c|c|c|c|}
\hline \multirow{2}{*}{\multicolumn{2}{|c|}{ Treatment }} & \multicolumn{4}{|c|}{2009 season } & \multicolumn{4}{|c|}{2010 season } \\
\hline & & \multicolumn{2}{|c|}{ Colour degree* } & \multicolumn{2}{|c|}{$\begin{array}{l}\text { Anthocyanine } \\
\text { content }\left(\mu \mathrm{g} / \mathbf{c m}^{2}\right)\end{array}$} & \multicolumn{2}{|c|}{ Colour degree } & \multicolumn{2}{|c|}{$\begin{array}{l}\text { Anthocyanine } \\
\text { content }\left(\mu \mathrm{g} / \mathrm{cm}^{2}\right)\end{array}$} \\
\hline \multirow[t]{2}{*}{$\begin{array}{l}\text { Thinning } \\
\text { out (To) }\end{array}$} & \multirow[t]{2}{*}{$\begin{array}{c}\text { Heading } \\
\text { back (HB) }\end{array}$} & $\begin{array}{c}\text { Fruit } \\
\text { thinning }\end{array}$ & $\begin{array}{l}\text { Fruit } \\
\text { thinning }\end{array}$ & $\begin{array}{c}\text { Fruit } \\
\text { thinning }\end{array}$ & $\begin{array}{l}\text { Fruit } \\
\text { thinning }\end{array}$ & $\begin{array}{c}\text { Fruit } \\
\text { thinning }\end{array}$ & $\begin{array}{c}\text { Fruit } \\
\text { thinning }\end{array}$ & $\begin{array}{c}\text { Fruit } \\
\text { thinning }\end{array}$ & $\begin{array}{c}\text { Fruit } \\
\text { thinning }\end{array}$ \\
\hline & & $\begin{array}{c}10 \mathrm{~cm} \\
(\mathrm{FT} 1)\end{array}$ & $\begin{array}{l}15 \mathrm{~cm} \\
\text { (FT2) }\end{array}$ & $\begin{array}{l}10 \mathrm{~cm} \\
(\mathrm{FT1})\end{array}$ & $\begin{array}{l}15 \mathrm{~cm} \\
(\mathrm{FT} 2)\end{array}$ & $\begin{array}{l}10 \mathrm{~cm} \\
(\mathrm{FT1})\end{array}$ & $\begin{array}{l}15 \mathrm{~cm} \\
(\text { FT2) }\end{array}$ & $\begin{array}{l}10 \mathrm{~cm} \\
(\mathrm{FT1})\end{array}$ & $\begin{array}{l}15 \mathrm{~cm} \\
(\mathrm{FT} 2)\end{array}$ \\
\hline \multirow{3}{*}{ To1 } & HB1 & 6.0 & 6.7 & 15.80 & 16.34 & 5.8 & 6.4 & 15.41 & 16.58 \\
\hline & HB2 & 7.1 & 8.0 & 15.89 & 17.25 & 6.9 & 7.8 & 16.07 & 17.01 \\
\hline & HB3 & 7.3 & 8.2 & 16.16 & 17.67 & 7.1 & 8.0 & 16.16 & 17.58 \\
\hline \multirow{3}{*}{ To2 } & HB1 & 6.6 & 7.1 & 15.98 & 16.52 & 6.3 & 6.9 & 15.94 & 16.75 \\
\hline & HB2 & 7.6 & 8.6 & 17.07 & 18.64 & 7.5 & 8.4 & 16.64 & 18.40 \\
\hline & HB3 & 7.8 & 8.5 & 16.81 & 18.46 & 7.6 & 8.3 & 16.46 & 18.00 \\
\hline \multirow{3}{*}{ To3 } & HB1 & 7.0 & 7.8 & 16.20 & 16.53 & 6.8 & 7.6 & 16.22 & 17.61 \\
\hline & HB2 & 7.9 & 8.5 & 17.18 & 18.34 & 7.6 & 8.3 & 17.13 & 18.24 \\
\hline & HB3 & 8.0 & 8.4 & 17.24 & 18.20 & 8.0 & 8.2 & 17.14 & 18.16 \\
\hline \multicolumn{2}{|c|}{ L.S.D. interaction 0.05} & \multicolumn{2}{|c|}{0.58} & \multicolumn{2}{|c|}{0.519} & \multicolumn{2}{|c|}{0.37} & \multicolumn{2}{|c|}{0.548} \\
\hline \multicolumn{2}{|c|}{0.01} & \multicolumn{2}{|c|}{0.79} & \multicolumn{2}{|c|}{0.695} & \multicolumn{2}{|c|}{0.77} & \multicolumn{2}{|c|}{0.737} \\
\hline \multirow{3}{*}{$\begin{array}{c}\text { Mean } \\
\text { effect of } \\
\text { thinning } \\
\text { out }\end{array}$} & $\begin{array}{c}\text { Thinning out } \\
25 \%\end{array}$ & \multicolumn{2}{|c|}{7.2} & \multicolumn{2}{|c|}{16.52} & \multicolumn{2}{|c|}{7.0} & \multicolumn{2}{|c|}{16.47} \\
\hline & $\begin{array}{c}\text { Thinning out } \\
50 \%\end{array}$ & \multicolumn{2}{|c|}{7.7} & \multicolumn{2}{|c|}{17.25} & \multicolumn{2}{|c|}{7.5} & 17 & 7.3 \\
\hline & $\begin{array}{c}\text { Thinning out } \\
75 \%\end{array}$ & & .9 & 17 & .28 & & 1.8 & 17 & .42 \\
\hline L.S. & D. 0.05 & 0 . & 24 & 0.2 & 211 & 0. & .23 & 0.2 & 224 \\
\hline L.S. & D. 0.01 & 0. & 32 & 0.2 & 284 & 0. & 31 & 0.3 & 300 \\
\hline $\begin{array}{c}\text { Mean } \\
\text { effect of }\end{array}$ & $\begin{array}{c}\text { Heading back } \\
0 \%\end{array}$ & 6 & .9 & & .23 & & .6 & 16 & .42 \\
\hline $\begin{array}{c}\text { heading } \\
\text { back }\end{array}$ & $\begin{array}{l}\text { Heading back } \\
25 \%\end{array}$ & 8 & .0 & 17 & .40 & & 1.8 & 17 & .25 \\
\hline & $\begin{array}{l}\text { Heading back } \\
50 \%\end{array}$ & & .1 & & .42 & & .9 & 17 & .25 \\
\hline L.S. & D. 0.05 & 0. & 24 & 0.2 & 211 & 0 . & 23 & 0.2 & 224 \\
\hline L.S. & D. 0.01 & 0. & 32 & 0.2 & 284 & & 31 & 0.3 & 300 \\
\hline $\begin{array}{c}\text { Mean } \\
\text { effect of }\end{array}$ & $\begin{array}{l}\text { Fruit thinning } \\
\qquad 10 \mathrm{~cm}\end{array}$ & & .3 & & 48 & & .1 & 16 & .35 \\
\hline $\begin{array}{c}\text { fruit } \\
\text { thinning }\end{array}$ & $\begin{array}{l}\text { Fruit thinning } \\
\qquad 15 \mathrm{~cm}\end{array}$ & & . 0 & & .55 & & 8.8 & 17 & .59 \\
\hline L.S. & D. 0.05 & 0. & 19 & 0.1 & 172 & & 19 & 0.1 & 183 \\
\hline L.S. & D. 0.01 & 0. & 26 & 0.2 & 232 & & .26 & & 246 \\
\hline
\end{tabular}




\section{References}

Abdel-Hamid, N. (1998) Effect of chemical thinning and thinning pattern on yield and fruit quality of Florida Prince. J. Agric. Sci., 7, 158-173.

Abo Ogiela, H.M.A. (2006) Response of guava trees to some pruning treatments and foliar application of potassium. M.Sc. Thesis, Fac. Agric., Kafr El-Sheikh, Tanta Univ., Egypt.

A.O.A.C. (Association of Official Agricultural Chemists) (1990) "Official Methods of Analysis", $15^{\text {th }}$ ed., Washington D.C., USA.

Attala, E.S. (1997) Effect of ways of hand thinning on Anna apple fruit quality and vegetative growth under Egy ptian desert conditions. Egypt. J. Agric. Res., 75, 1081-1096.

Bussi, C., Lescourret, F. and Ganard, M. (2009) Effect of thinning and pruning on shoot and fruit growth of girdled fruit-bearing shoots in two peach cultivars (Big Top and Alexandra). Europ. J. Hort Sci., 74 (3), 97-102.

Corelli-Grappadelli, L. and Costen, D.C. (1991) Thinning pattern and light environment in peach tree canopies influence fruit quality. HortScience, 26, 1464-1466.

Davarynefad, G.H., Nyéki, J., Szabó, T. and Szabó, Z. (2008) Influence of hand thinning of buds and blossom on crop load fruit characteristics and fruit growth dynamic of Úifehértói Fürtös sour cherry cultivar. American Eurasian J. Agric. \& Environ. Sci., 4 (2), 138-141.

Demirtas, M.N., Bolat, I., Ercisle, S., Lkinci, A., Olmez, H.A., Sahin, M., Altindag, M. and Celik, B. (2010) The effect of different pruning treatments on the growth, fruit quality and yield of "Hacihaalilglu" apricot. Acta Sci. Pol. Hortorum Cultus, 9 (4), 183-192.

Egea, J., Berengue, T., Egea, L. and Garcia, J.E. (1989) Adaptation of peach cultivars to warm winters. Anales de Edafulogiay Agrobiologia, 48, 205-217.

Eliwa, G.I. (2003) Effect of gridling and fruit thinning on maturity, yield and fruit quality of "Mit Ghamr" peach trees. Egypt. J. Hort., 30 (3-4), 281-290.

Fumey, D., Louri, P.E., Guedon, Y., Godin, C. and Costes, E. (2008) Effect of pruning on the apple trees: from tree architecture to modeling. Inra, Equipe Architecture 3, 1-6.

Hsia, C.L., Luh, B.S. and Chicherter, C.O. (1965) Anthocy anin in frestone peaches. $J$. Food Sci., 30, 5-12.

Li, K.T., Lukso, A., Piccioni, R. and Robinson, T. (2003) Summer pruning affects on fruit size, fruit quality, return bloom and fine root survival in apple trees. Journal of Horticultural Science \& Biotechnology, 78 (5), 755-761.

Mahajan, B.V.C. and Dhillon, B.S. (2002) Effect of pruning intensities on the fruit size, yield and fruit quality of peach cv. Shan Punjab. Agric. Sci. Digest 22 (4), 281-282. (C.F. Recor 211 of 252 CAB Abstracts, 2003/11-20-04/07).

Egypt. J. Hort. Vol. 42, No.1 (2015) 
Mansour, N.M. and Stino, G.R. (1986a) Growth and flowering behaviour of some American peach cultivars under Egy ptian climate. Agric. Res. Rev., 64 (3), 397-411.

Mansour, N.M. and Stino, G.R. (1986b) "Early Grand" and "Desert Gold", two promising peach cultivars adapted to Egypt. Agric. Res. Rev., 64 (3), 412-424.

Mika, A. (1986) Phy siological responses of fruit trees to pruning. Hort. Rev., 8, 337-378.

Mikhael, G.B. (2001) Effect of some agricultural treatments on growth and yield as related to alternative bearing of Japanese persimmon. Ph.D. Thesis, Fac. of Agric., Kafr El-Sheikh, Tanta Univ., Egypt.

Mikhael, G.B., Omar, A.K. and Gabr, M.A. (2012) Yield and fruit quality of "Dessert Red" peach trees as influenced by planting density and pruning severity. J. Biol. Chem. Environ. Sci., 7 (2), 123-144.

Ministry of Agriculture and Land Reclamation (2013) Agricultural Economics. Annual Report, Cairo, Egy pt.

Mohsen, A.T. (2010) Thinning time and fruit spacing influences on maturity yield and fruit quality of peaches. J. Hort. Sci. \& Ornamm. Plants, 2 (3), 79-87.

Myer, S.C., King, A. and Savelle, A.T. (1993) Bloom thinning of Winble peach and Fantasia nectarine with monocarbamide dihy drogen-sulfate. HortScience, (28) 616-617.

Njorog, S.M.C. and Reighard, G.C. (2008) Thinning time during stage 1 and fruit spacing influence fruit size of "Contender" peach. Scientia Horticulturae, 15, 352-359.

Rathi, D.S., D.C. Dinri, M.C. Noutiyol and Kumar (2003) Pruning response to shoot growth, fruit set and yield in peach. India. J. Hort., 60 (2), 151-153.

Reginato, M.G., Errazuris, E.R. and Camus, C.J.L. (1995) Evaluation of intensity of crop thinning in nectarines by measuring unit fruit number $/ \mathrm{cm}^{2}$ of trunk. Agric. Tecnia, 55, 42-47.

Said, I.A., Eissa, F.M. and Kandil, E.A. (2003) Effect of winter pruning, hand thinning and girdling on "Canino" apricot growth yield and quality. Minia J. of Agric. Res. \& Develop, 23 (2), 301-328.

Samra, N.R., Mansour, A.M., El-Dengaw, E.F. and Tarabih, M.E. (2002) Influence of studies on thinning and girdling on apple fruit quality. $2^{\text {nd }}$ Inter. Conf. Hort. Sci. Kafr El-Sheikh, Tanta Univ., 28 (3), 1256-1265.

Sansavini, S., Corelli, L. and Giunchi, L. (1985) Peach yield efficiency as related to tree shape. Acta. Hort., 173, 139-158.

Sharma, D.P., Chauhan, J.S. and Sharma, N. (2001) Response of different pruning intensities and fertilization treatments on fruit grading and maturity of peach (Prunus persica Batsch) cv. July Elberta. Praressive Hort., 33 (2), 204-207. 
Siham, M., Bussi, C., Lescourret, F., Genard, M., Habib, R. and Gilreath, J. (2005) Pruning intensity and fruit lead influenced on vegetative and fruit growth in Alexandria Peach. Proc. Fla. State. Hort. Soc., 118, 266-269.

Snedecor, G.W. and W.G. Cochran (1990) "Statistical Methods" $7^{\text {th }}$ ed. The Iowa State Univ. Press, Ames. Iowa, USA, 593 p.

Southwick, S.M., Weis, K.G., Yeager, J.T. and Zhou, H. (1995) Controlling cropping in "Lodel Cling" peach using gibberellin effects on flower density fruit distribution, fruit firmness, fruit thinning and yielding. J. Amer. Soc. Hort. Sci., 120, 1087.

Stino, R.G. (1995) Effect of type and time of dormant pruning on blooming, fruiting and leaf $\mathrm{Fe}, \mathrm{Zn}$ and $\mathrm{Mn}$ content of LeCont pear trees. Egypt. J. Hort., 22 (2), 221-242.

Zayan, M.A., Morsy, E., Ayead, H.M. and Gaber, M.A. (2002) Influence of pruning treatments on growth, leaf constituents, flowering, yield and fruit quality of "Anna:" apple trees. I. Effect of dormant pruning treatments. $2^{\text {nd }}$ Inter. Conf. Hort. sci. Kafr ElSheikh, Tanta Univ., 28 (3), 1203-1228.

Zayan, M.A. (1991) Vegetative growth, yield and fruit quality of "Mit-Ghamr" peach trees as influenced by: 1-pruning severity. J. Agric. Res., Tannta Univ., 17 (3), 658667. 


$$
\begin{aligned}
& \text { تحسين إنتاجية محصول وجودة أثجار الخوخ صنف فلوريدا برنس } \\
& \text { بإستخدام بعض المعاجة معرلات والزراعية } \\
& \text { محمد عبده زيان ، سمير محمد زعربان ، جهاد بشرى يوسف ميخائيل" }
\end{aligned}
$$

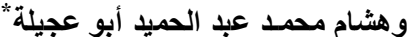

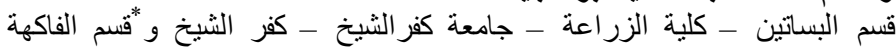

$$
\begin{aligned}
& \text { متساقطة الأوراق - معهد بحوث البساتين - مركز البحوث الزراعية ـ القاهرة - }
\end{aligned}
$$

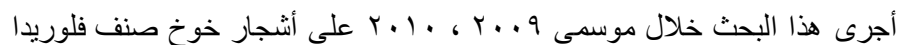

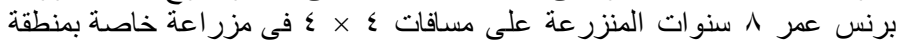

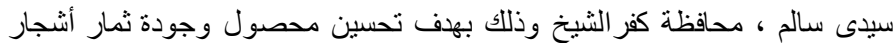

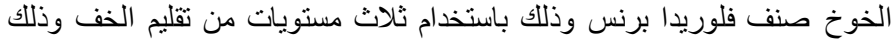

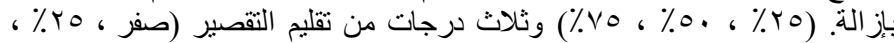

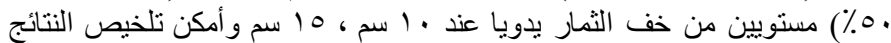
الكتحصل عليها فى النقاط الآتية:

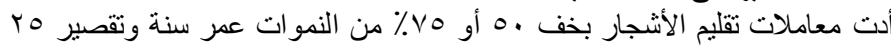

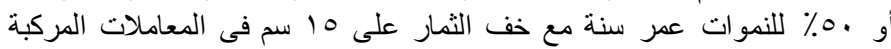

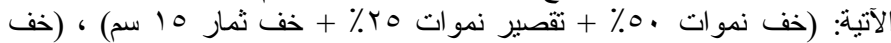

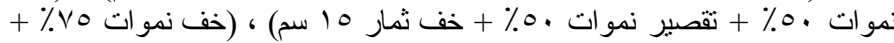

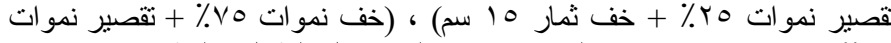

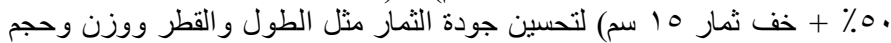

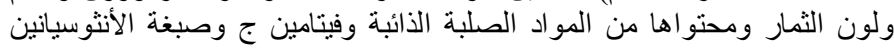

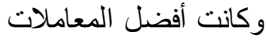

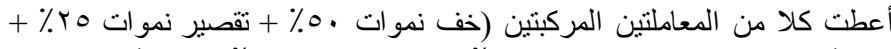

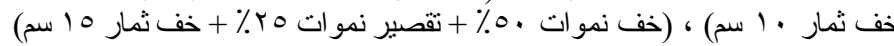

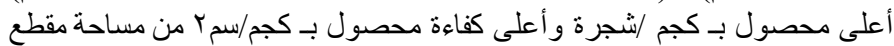

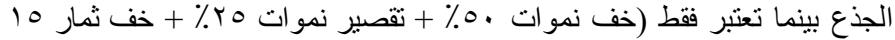

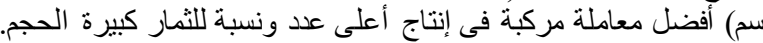

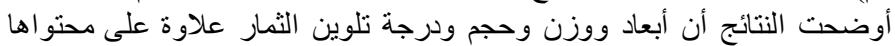

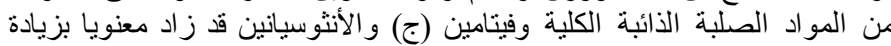
شدة تقليم الخف و التقصير ودرجة خف الثة الثمار

لذلك يوصى هذا البحث مزارعى الخوخ صنف الفلوريدا برنس بتقليم أشجار هم

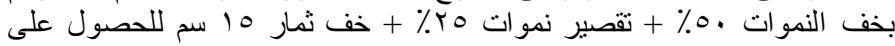

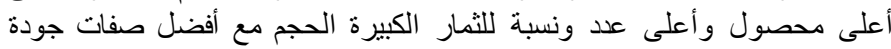
خاصة ووزن وحجم وتلوين الثمار ومحتواها من المواد الصلبة الذائبة الكلية الكاتية و الأنثوسيانين. (TSS) 